\title{
Die Placenta praevia-Therapie an der Kgl. Universitäts-Frauenklinik zu Miinchen in den letzten 10 Jahren (1907-1917).
}

\author{
Von \\ Dr. LudWig Nürnberger, \\ Assistent der Klinik.
}

"Häufig ist in der Medizin die Theorie sehr einfach und die praktische Erfüllung der Aufgabe riesig schwer." Diese Worte Zweifel's ${ }^{1}$ ) wird man wohl noch Jahrzehnte lang jeder Erörterung über die Behandlung der Placenta praevia als Motto mit auf den Weg geben können.

Die Theorie der Placenta praevia hat in den letzten Jahren wesentliche Fortschritte gemacht.

Seit langer Zeit spielte in der Lehre von der Aetiologie der Placenta praevia die „Endometritis" eine grosse Rolle. Zur Annahme eines derartigen Zusammenhangs führte einesteils die Tatsache, dass bei Mehrgebärenden Placenta praevia weitaus häufiger vorkommt als bei Erstgebärenden, weiter der Umstand, dass sich in der Anamnese Placenta praevia-kranker Frauen häufig Anhaltspunkte für pathologische Veränderungen des Endometriums (Menstruationsstörungen, Fluor, vorausgegangene Aborte, manuelle Plazentarlösungen u. dgl.) fanden. Ueber den näheren kausalen Zusammenhang zwischen den supponierten endometrischen Veränderungen und der heterotopen Insertion des Eies konnte man sich freilich keine so recht klaren Vorstellungen machen. Hier war es nun $\mathrm{Hoehne}^{2}$ ), der durch seine ausgedehnten und sorgfältigen Untersuchungen über die Flimmerung im weiblichen Genitale die korrelativen Beziehungen zwischen abnormer Nidation des Eies und Beschaffenheit der Uterusinnenfläche unserem Verständnis näher gerückt hat. Hoehne fand, dass bei geschlechtsreifen Frauen der Uterus unter normalen Verhältnissen im Gegensatz zur Tube stets eine lückenhafte, diskontinuierliche Flimmerung zeigt. Bei chronisch-hyperplastischen Zuständen der Korpusmukosa findet sich dagegen im Uterus eine abnorm ausgebreitete und abnorm starke Flimmerung, so dass ein wirklich kontinuierlicher Flimmerstrom entstehen kann. Trifft nun das befruchtete $\mathrm{Ei}$ auf eine solche hyperplastische Schleimhant mit über-

1) Döderlein's Handb. d. Geburtsh. Bd, 2. S. 593.

2) Hoehne, Zentralbl. f. Gyn. 1908. S. 121 u. 1911. S. 340. 
mässig entwickeltem, zu stark arbeitendem Flimmerstrom, so kommt es in den oberen Partien des Korpus nicht zur Ruhe, sondern es wird weiter nach unten getrieben. Siedelt es sich dann in den unteren Partien des Uterus an, so ist damit der Grund zur Entstehung einer Placenta praevia gelegt. Auf ein weiteres ätiologisch wichtiges Moment hat R. Meyeri) hingewiesen. Es ist eine allgemein anerkannte und bei Tieren zur Genüge erwiesene Tatsache, dass sich das befruchtete Ei nicht zu irgend welchem Zeitpunkte implantiert, sondern nur, wenn es eine ganz bestimmte Stufe seiner Entwicklung, die sog. Nidationsreife (R. Meyer) erreicht hat. Ferner darf als erwiesen gelten, dass sich das befruchtete Ei, wenn es dieses Reifestadium erreicht hat, implantieren kann," wo es sich gerade befindet, im Ovarium, in der Bauchhöhle, in der Tube, im Uterus. Für gewöhnlich sind der Befruchtungsort und das Tempo der Eifortbewegung so aufeinander eingestellt, dass sich das Ei zur Zeit seiner Einnistungsfighigkeit gerade im Corpus uteri befindet. Wandert es dagegen unter dem Einfluss irgend welcher Faktoren so schnell, dass es bereits im Cavum nteri anlangt, bevor es zur Nidation reif ist, so wird es durch die Flimmerung weiterbefördert und nistet sich erst in einer tieferen Partie des Uterus ein. Auch hierdurch sind also die Vorbedingungen zur Entstehung einer Placenta praevia gegeben.

Ebenso wie die Lehre von der Genese der Placenta praevia haben auch die Ansichten über ihr anatomisches Verhalten zur Uterrus: wand durch die Untersuchungen der letzten Jahre eine Vertiefung und Klärung erfahren. Die ganze Lehre von der heterotopen Okkupation des unteren Uterusabschnittes durch das $\mathrm{Ei}$ steht in engem Zusammenhang mit der Frage, ob dieser untere Uterusabschnitt in anatomischer und funktioneller Hinsicht eine Sonderstellung einnimmt oder nicht. Durch die Untersuchungen A schoff's ${ }^{2}$ ) und seiner Schüler [Hohmeier ${ }^{3}$ ), K. Hegar $\left.\left.\left.{ }^{4}\right) \cdot 0 \operatorname{gata}^{5}\right)\right]$ wurde nun der einwandfreie Beweis erbracht, dass die alte Lehre von der Zweiteilung des Uterus in das kontraktionsfähige. Korpus und in die kontraktionsunfähige, nur als Ausführungsgang dienende, Zervix nicht richtig ist, sondern dass am graviden wie am nichtgraviden Uterus zwischen Korpus und Zervix ein dritter, anatomisch und funktionell wohl charakterisierter, selbståndiger Abschnitt, der sog. Isthmus eingeschaltet ist. Die obere Grenze, des Isthmus, das Orificium internum anatomicum Aschoff's, entspricht dem früheren „inneren Muttermund“ der Autoren. Sie ist schon äusserlich durch eine leichte Einschnürung und in der Regel durch die Abgangsstelle der vorderen Umschlagsfalte des Peritoneums gekennzeichnet. Die untere Grenze des Isthmus, das Orificinm internum histologicum Aschoff's, wird gebildet von der scharfen Grenze der Isthmus- gegen die Zervixschleimhaut. Die Schleimhaut des Isthmus unterscheidet sich von der Mukosa des Corpus uteri nur durch die geringe Dicke, durch ihre unregelmässigere Begrenzung gegen die Muskularis und durch den spärlicheren Gehalt an Drüsen. Diese

1) R. Meyer, Zentralbl. f. Gyn. 1911. S. 538.

2) Aschoff, Verhandl. d. Deutschen pathol. Ges. Kiel 1908.

3) Hohmoier, Monatsschr. f. Geb. u. Gyn. Bd. 22.

4) K. Hegar, Beitr. z. Geb. u. Gyn. Bd. 13.

5) Ogata, Beitr. z. Geb. u. Gyn. Bd. 13. 
verlaufen in der Regel von unten aussen nach oben innen. Die Mus-. kulatur des Isthmus ist lockerer gefügt und reicher an Bindegewebe als die des Korpus. Sie weist ferner zahlreiche grosse Gefässstämme auf, da ja in der Gegend des Isthmus die Uterina an den Uterus herantritt.

Nachdem so der Isthmus als ein autonomer Abschnitt des Uterus erkannt war, handelte es sich darum, seine Rolle in der Physiologie und Pathologie der Eiansiedlung festzustellen. Die Klärung dieser Verhältnisse verdanken wir Pankow ${ }^{1}$ ). Zunächst konnte Pankow zeigen, dass sich der Isthmus in jeder Schwangerschaft entfaltet und durch Verwachsung seiner Schleimhaut mit den Eihäuten zur Bildung: der Eikammer verwendet wird. Weiter kounte Pankow aber auch durch den Nachweis der Durchsetzung der Isthmuswand mit chorialen Elementen den einwandfreien. Beweis erbringen, dass eine primäre Insertion des Eies im Isthmus erfolgen kann.

Aber nicht nur der Isthmus, sondern auch die Zervix kann, wie Beobachtungen und Untersuchungen der letzten Jahre gezeigt haben, bei abnorm tiefem Sitz des Eies in mehr oder minder hohem Grade der Plazentation dienen. Die Grenze zwischen der Korpusschleimhaut des Isthmus und der Zervixsschleimhaut bildet für die Ausbreitung der Chorionzotten also durchaus nicht eine so starre Barriere, wie man lange Zeit geglaubt hatte. Nachdem wir ${ }^{2}$ ) im Jahre 1914 im Anschluss an einen eigenen Fall von isthmisch-zervikaler Schwangerschaft zwölf Beobachtungen von Insertion der Plazenta im Zervixkanal aus der Literatur zusammenstellen konnten, die sich über einen Zeitraum von 27 Jahren verteilen, sind seitdem, also in einem Zeitraum von etwas mehr als 3 Jahren, allein 3 Fälle von Zervixplazenta, einer von Jaschke ${ }^{3}$, einer von Gertrud Tiegel ${ }^{4}$ ) und einer von Vogt5) veroffentlicht, worden. Sicher hat Jaschke recht, wenn er darauf hinweist, dass man bei genauer Untersuchung eine partielle 0kkupation der Zervix durch Plazentargewebe nicht allzu selten finden wird.

Die Erweiterung unserer Kenntnisse über die pathologisch-anatomischen Vorgänge bei heterotoper Insertion des Eies ist von weittragender praktischer Bedeutung.

Durch die Nidation in Isthmus kommt das Ei in unmittelbare Nähe des Stammes der Uterina und ihrer grossen Aeste. Schon hieraus lassen sich gewisse bei Placenta praevia häufige Erscheinungen erklären. So erscheint eś uns wahrscheinlich, dass ein Teil der Aborte bei tiefem Sitz des Eies darauf zurückzuführen ist, dass bei der Bildung der intervillösen Räume das aus den grossen Uterinaästen unter hohem Druck einschiessende Blut den Plazentarhaftboden unterwühlt und, zu mehr oder minder weitgehender Ablösung des Eies führt. Ferner erklären sich aus der Nähe der grossen Gefässe die oft so foudroyanten Schwangerschaftsblutungen, ferner die Blutungen unter der Geburt und die Nachgeburtsblutungen.

Hierzu kommt aber noch ein weiterer. Umstand. Aus noch nicht

1) Pankow, Beitr. z. Geb. u. Gyn. Bd. 15 u. 16.

2) Nürnberger, Prakt. Ergebn. d. Geb. u. Gyn. Bd. 6.

3) v. Jaschke, Zentralbl. f. Gyn. 1917. S. 1065.

4) G. Tiegel, Zeitschr. f. Geb. u. Gyn. Bd. 77. S. 399

5) Vogt, Deutsche med. Wochenschr. 1917. S, 1041. 
näher geklärten Gründen — vielleicht infolge der geringen Dicke der Schleimhaut oder infolge der beschränkten Möglichkeit einer Ausdehnung in der Fläche - kommt es in vielen Fällen von tiefem Sitz der Plazenta zum abnormen Vordringen fötaler Elemente in die Tiefe. Hierbei kann es sich nur um das Eindringen synzytialer Zellen (Pankow, Nürnberger) handeln, oder aber es kann zu mehr oder minder hochgradiger Zerstörung der ganzen Uteruswand durch das Einwuchern von Chorionzotten kommen [R. Meyer ${ }^{1}$ ), M. B. Schmidt ${ }^{2}$ ), v. Jaschkè ${ }^{3}$ ), Tiegel4), Vogt5)]. Das Eindringen von Trophoblastzellen kann dann noch sekundär zu Degenerationserscheinungen an der Muskulatur führen (Nürnberger, Vogt). Schon allein das rein mechanische Moment der Auseinanderdrängung der kontraktilen Substanz durch ortsfremde Elemente muss anf die ganze Dynamik, speziell die Blutstilluag in der Nachgeburtsperiode von Einfluss sein. Hierzu kommen dann auch noch die chemischen Schädigungen durch die invasiven fötalen Elemente. Hierdurch erklären sich leicht und ungezwungen nicht nur die auffallende Brüchigkeit und Morschheit der von der Plazenta besiedelten Uteruspartien, sondern auch die oft jeder Therapie trotzenden enormen Blutungen unter der Geburt und in der Nachgeburtsperiode.

Für die Praxis ergeben sich hieraus verschiedene wichtige Folgerungen. Zunächst ist zu fordern, dass jede Placenta praevia-Kranke möglichst bald und rasch in klinische Verhältnisse gebracht wird, da nur hier eine ausreichende, sichere Blutstillung gewährleistet ist. Weiter sind in der Behandlung der Placenta praevia die Methoden zu bevorzugen, welche durch möglichst baldige Beendigung der Geburt allzugrosse Blutverluste vermeiden und eine sichere Blutstillung garantieren. Endlich soll man sich bei starken Blutungen in der Nachgeburtsperiode nicht allzulange mit den gewöhnlichen Mitteln der Blutstillung aufhalten, sondern gegebenenfalls auch vor der Totalexstirpation des Uterus nicht zurïckschrecken ${ }^{6}$ ).

Neben der Blutung* findet sich als weitere, durchaus nicht séltene Komplikation der Placenta praevia die abnorme Adhärenz des Mutterkuchens, die ihr Extrem in der festen, völlig unlösbaren Verbindung von Plazenta und Uteruswand, der sog. Placenta praevia accreta erreicht. Dass bei Placenta praevia Störungen der Nachgeburtsperiode durch abnorm festes Haften des Mutterkuchens auf seiner Unterlage häufig sind, ist eine alte Erfahrung.

Ueber die feineren, dieser Erscheinung 'zugrunde liegenden Vorgänge wusste man bis vor kurzer Zeit noch so gut wie nichts. Heute wissen wir, dass dem klinischen Bild der Placenta accreta überhaupt kein einheitliches anatomisches Substrat entspricht.

1) R. Meyer, Zeitschr. f. Geb. u. Gyn. Bd. 70. S. 334.

2) M. B. Sohmidt, Ziegler's Beitr. z. pathol. Anat. u. allg. Pathol. Bd. 63. S. 285.

3) v. Jaschke, Zeitschr. f. Geb. u. Gyn. Bd. 67. S. 656 .

4) Tiegel, l.c.

5) $\operatorname{Vog} t, 1, c$.

6) Auch die von $\mathrm{Z}$ weifel (Doederlein's Handbuch d. Geburtsh. Bd. 2. S. $613 \mathrm{ff}$.) angegebene Tamponade mit säurefreiem Liquor ferri kann — so gut sie sich uns sonst bewährt - bei Blutungen aus grossen Uterinaästen versagen. 
Der erste einwandfrei und genau untersuchte Fall von pathologischer Adhärenz einer Placenta praevia stammt, von $\mathrm{Bais} \mathrm{ch}^{1}$ ). In diesem Falle fehlte an der Decidua basalis jede Andeutung einer spongiösen Schicht. Auch am Myometrium fanden sich Veränderungen, vor allem eine ausgedehnte bindegewebige Durchsetzung.

Dieser Beobachtung von Baisch konnten wir selbst ${ }^{2}$ ) eine weitere ebenfalls vom Ende der Schwangerschaft anfügen. In unserem Fall, von Placenta praevia accreta fanden sich neben den schon von Baisch beschriebenen Veränderungen an der Dezidua und dem Bindegewebsapparat eine ausgedehnte Durchsetzung des Myometriums mit chorioepithelialen Elementen sowie sekundäre degenerative Veränderungen an den Muskelfasern.

In einer weiteren Reihe von Fällen (R. Meyer, M. B. Schmidt, Vogt, Tiegel) war die abnorme Adhärenz einer Placenta praevia darauf zurückzuführen, dass eine Dezidua völlig fehlte und die Chorionzotten an oder in der Muskulatur inserierten. Ferner war in einigen dieser Fälle die Plazenta in ganz diffuser Weise und an ausgedehnten Stellen in die Muskulatur vorgedrungen, so dass es nicht nur zu höchstgradiger pathologischer Adhärenz, sondern auch zu ausgedehnter Rarefizierung und Destruktion grosser Teile der Uteruswand gekommen war.

Die klinische Bedeutung der abnormen Adhärenz einer Placenta praevia ist nicht gering. Auf die Gefahr durch abundante Blutungen haben wir schon oben hingewiesen. Weiter kommt hinzu, dass die zurückgebliebenen, oft sehr bedeutenden Plazentarreste die Eingangspforte für Infektionen bilden können, die nach Lage der ganzen anatomischen Verhältnisse in der. Regel viel schwerer verlaufen, als beim Sitz der Plazenta im Korpus. Auch für solche Fälle von hochgradiger Adhärenz einer Placenta praevia möchten wir daher als Regel aufstellen, mit der totalen Entfernung des sowieso schon schwer geschädigten Uterus nicht allzulange zu zögern.

Die Bereicherung unserer Kenntnisse über die Ursachen und die Entstehung der Placenta praevia hat nicht zu einer Konvergenz der therapeutischen Richtlinien geführt, sondern sie hat eher das Gegenteil bewirkt. Neue Methoden sind zu den alten hinzugekommen, ohne dass diese wertlos oder überflüssig geworden wären, und so gibt es heute nur wenige geburtshilfliche Eingriffe, die nicht bei Placenta praevia angewendet werden könnten, ja gelegentlich angewendet werden müssten.

Von den zahlreichen Wegen, die heute für die Placenta praevia-Therapie gangbar sind, muss einer der beste sein. Welcher es ist, lässt sich zurzeit noch nicht entscheiden. Dazu bedarf es grosser Statistiken über viele Tausende von Fällen. Schon aus diesem Grunde dürfte die Mitteilung von einer grösseren Reihe von

1) Baisch, Arbeiten aus dem pathol. Institut zu Tübingen. 1908. Bd. 6. H. 2. S. 265.

2) Nürnberger, Prakt. Ergebn. d. Geb. u. Gyn. Bd. 6. H. 1. 
Placenta praevia - Fällen und der bei ihrer Behandlung erzielten Resultate nicht ganz uninteressant sein.

Hierzu kommt aber noch, dass die Münchener UniversitätsFrauenklinik unter Döderlein's Leitung den vaginalen Kaiserschnitt als die beste Behandlungsmethode gegenüber allen anderen Verfahren bevorzugt. Es werden die nachfolgenden Zeilen zum grossen Teil also der Frage des vaginalen Kaiserschnittes in der Placenta praevia-Behandlung gewidmet sein.

In der Zeit vom 1. X. 1907 bis 1. X. 1917, also in einem Zeitraum von 10 Jahren, ereigneten sich in der Münchener Universitäts-Frauenklinik 27980 Geburten. Unter diesen $27980 \mathrm{Ge-}$ burten waren nun 234 Fälle $=0,83$ pCt. von Placenta praevia, d. h: auf 119 Geburten traf eine Placenta praevia.

Diese Zahl hat nun insofern einen relativen Wert, als sie eben nichts weiter bedeutet, als die Häufigkeit der Placenta praeviaFälle in der Münchener Universitäts-Frauenklinik. Eine ganz spezifische Note erhält sie aber durch folgende Ueberlegung: Die absolute Häufigkeit der Placenta praevia wird auf Grund grosser Länderstatistiken. [Döderlein ${ }^{1}$ ), $\vee$. Seuffert2)] als durchschnittlich 1:500-600 angenommen, d. h. auf etwa 500-600 Geburten trifft 1 F'all von vorliegender Nachgeburt. Die Geburtenzahl in München betrug nun für das Jahr 191313169 Kinder. Nehmen wir das Verhältnis 1 Placenta praevia: 500 Geburten an, so würde dies für München 26 Fälle von Placenta praevia ergeben. Tatsächlich wurden im Jahre 1913 aber 29 Fälle von Placenta praevia in die Königliche Universitäts-Frauenklinik eingeliefert. Von diesen stammen 23 aus München und 6 von auswärts. Es sind demnach nahezu sämtliche Fälle von Placenta praevia, die sich im Jahre 1913 in München ereigneten, der Königlichen Universitäts-Frauenklinik zur Behandlung überwiesen worden. Aehnliche Verhältnisse lassen sich auch für die übrigen Jahre berechnen. Diese Tatsache beweist, dass der schon wiederholt von versehiedensten Seiten aufgestellten Forderung der klinischen Behandlung von Frauen mit Placenta praevia in München weitgehend nachgekommen wird.

1) Döderlein, Ueber die Behandlung der Placenta praevia. Verhandl. d. 17. intern. med. Kongr. London 1913.

2) Dödeŕlein-Krönig, Operative Gynäkologie. 3. Aufl. S. 922. 


\section{Mütterliche Mortalität.}

Von den 234 an Placenta praevia in der Münchener Universitäts-Frauenklinik behandelten Frauen sind insgesamt 21 gestorben. Das bedeutet also eine mütterliche Gesamtmortalität von 8,9 pCt. Diese Zahl steht in fast vollkommener Uebereinstimmung mit dem Werte $(8,05 \mathrm{pCt}:)$, den Döderlein $\left.{ }^{1}\right)$ aus seiner grossen internationalen klinischen Statistik von 5615 Fällen für die mütterliche Sterblichkeit berechnete.

Bei der Wertung dieser Zahl sind verschiedene Punkte zu berücksichtigen. Vor allem muss man sich vergegenwärtigen, dass die $8 \mathrm{pCt}$. mütterliche Mortalität nur für die Kliniken gelten. Die Geburtshilfe des Privathauses muss mit einer weit höheren Sterblichkeit der Placenta praevia-Fälle rechñen, nämlich mit rund 19 pCt. (Döderlein, v. Seuffert, Füth). Die Gegenüberstellung dieser Zahl zeigt zur E Evidenz, dass die Sterblichkeit der Mütter bei klinischer Behandlung um mehr als die Hälfte geringer ist als die allgemeine Mortalität bei Placenta praevia.

Aber trotzdem sind die $8,9 \mathrm{pCt}$. Mortalität immer noch relativ hoch.

Bei der kritischen Sichtung unserer 21 Todesfälle ist zunächst eine Frau mit einer hochfloriden, offenen Lungentuberkulose zu erwähnen. Im Auswurf fanden sich reichlich Tuberkelbazillen. Die Kranke kam schon mit einer Temperatur ron 39,10 in die Klinik und erlag am 6. Tage post partum einer schweren Hämoptoe.

Nach Abzug dieses einen Falles beträgt unsere mütterliche Mortalität noch $8,5 \mathrm{pCt}$.

Von den noch verbleibenden 20 Frauen sind 14, d. i. mehr als die Hälfte an Anämie zugrunde gegangen, 6 erlagen einer Sepsis.

Die Beteiligung der einzelnen bei Placenta praegia in Betracht kommenden Eingriffe an der mütterlichen Mortalität wird bei der Besprechung der verschiedenen Methoden erörtert werden. Hier sei zunächst nur erwähnt, dass sich in unserem Material auch zwei Todesfälle nach Spontangeburt finden ${ }^{2}$ ) Die eine Patientin erlag einer Anämie, die andere einer Sepsis.

1) 1. $\mathrm{c}$.

2). Bei einer dritten Frau (1908 G. N. 1023), die in der Klinik spontan niederkam, war'draussen die Blase gesprengt worden. Die näheren Einzelheiten dieses Falles finden sich bei der Blasensprengung erwähnt. 
In dem ersten Fall (1915 A. N. 2557) handelte es sich um eine 37jährige II. Gebärende, die nach Angabe ihrer Angehörigen schon draussen in ihrer Wohnung einen sehr starken Blutverlust erlitten hatte. In der Klinik selbst blutete die ziemlich anämische Pat. nicht mehr und es erfolgte hier alsbald die Spontangeburt eines lebenden, reifen Mädchens. Die Plazenta kam auf Credé. Einige Minuten später trat eine sehr starke Atonie mit einer foudroyanten Blutung auf: Ausgiebige Uterusscheidentamponade. Diese wurde schon nach kurzer Zeit wieder durchblutet und unter den Zeichen der Herzschwäche erfolgte 1/2 Stunde post partum der Exitus.

In dem zweiten Falle (1917 G. N. 976) wurde die Pat. - eine XI. Gebärende im 7. Monat der Schwangerschaft - tamponiert aus der Poliklinik eingeliefert. Die Kranke hatte draussen sehr stark geblutet, und auch bei der Aufnahme in die Klirik sickerte das Blut schon wieder durch die Tamponde hindurch. Die Frau sah sehr anämisch aus, Puls 116, auch bestand schon leichtes Fieber $\left(37,9^{\circ}\right)$. In der Klinik stand auf neue, feste Tamponade die Blutung vollkommen. Einige Stunden später spontaner Blasensprung bei noch liegender Tamponade. Es setzten nun kräftige Wehen ein, einige Minuten später wurde die Tamponade spontan ausgestossen und der Steiss des Kindes in der Vulva sichtbar. Das Kind kam spontan bis auf den Kopf, der mittels des Veit-Smellie'schen Handgriffes entwickelt wurde. Wegen Blutung wurde die Plazenta manuell gelöst. Am Abend stieg die Temperatur auf $39,7^{\circ}$ und hielt sich in der Folgezeit unter späteren morgendlichen Remissionen auf dieser Höhe. 2 Tage nach der Entbindung wurden aus dem Blute schon Streptokokken gezüchtet und am 12. Tage nach der Niederkunft erfolgte unter den Zeichen der Sepsis der Tod.

Für den Verblutungstod jedes klinisch behandelten Placentai praevia-Falles kommen zwei Momente in Betracht, nämlich:

1. der ausserhalb der Klinik,

2. der innerhalb der Klinik, speziell bei der Entbindung, erlittene Blutverlust.

Der schliessliche Ausgang jedes in eine Anstalt verbrachten und an Verblutung gestorbenen Falles von vorliegender Nachgeburt. ist eine algebraische Funktion dieser beiden Grössen. Hierbei ist. der bei der Eatbindung auftretende Blutverlust eine unabhängige Variable. in dem Sinne, dass eben mit einem gewissen Blutverlust. bei der Entbindung immer gerechnet werden muss. Freilich wird sich dieser je nach der Entbindungsart, der Technik des Operateurs usw. verschieden gross gestalten. Ueberschreitet der Blutverlust, den die Patientin bis zu ihrer Entbindung erlitten hat, ein gewisses Mass, dann wird auch das schonendste und schnellste Entbindungsverfahren den unglücklichen Ausgang nicht verhindern können. 
Bei allen unseren verbluteten Frauen findet sich nun, wie aus den später bei den einzelnen Behandlungsmetboden erwähnten Krankengeschichten zu ersehen ist, ohne Ausnahme in den Journalen vermerkt, dass die Patientinnen sich bei ihrer Einlieferung in die Klinik in schwer ausgeblutetem Zustande befanden. Einige wurden sogar fast pulslos eingeliefert.

In der Klinik selbst war der Blutverlust gering. Nur bei einer (1910 S. A. 52) betrug er $1000 \mathrm{~g}$. Hier ist aber der Blutverlust nach der Geburt sehuld. Die Kranke durchblutete infolge einer schweren Atonie zweimal die ganze Uterusscheidentamponade. Bei einer anderen Patientin (1913 A. N. 19170) wurde sogar wegen der heftigen Atonie die Totalexstirpation gemacht. Freilich konnte auch diese den letalen Ausgang nicht mehr aufhalten.

Wenn wir eingangs mit einer gewissen Genugtuung darauf hinweisen konnten, dass in München die weitaus überwiegende Mehrzahl aller Placenta praevia-Fälle der Klinik überwiesen wird, so heisst das allerdings nur, dass diese Fälle im Privathause nicht beendet, nicht aber, dass sie dort nicht behandelt wurden. Die 14 an Anämie zugrunde gegangenen Patientinnen zeigen aber, dass die Einweisung einer Placenta praevia-kranken Frau in eine Anstalt allein noch nicht genügt, sondern dass sie auch rechtzeitig erfolgen muss. Gewiss wäre es unrecht, für alle in der Klinik ad exitum gekommenen Fälle die Aerzte draussen verantwortlich zu machen; gewiss können, man kann fast sagen auf dem Operationstiseh der Klinik noch, foudroyante Blutungen auftreten. Einige Fälle aber, in denen uns schwer anämische Frauen eingeliefert wurden, bei denen die Aerzte draussen vergebens die Wendung nach Braxton-Hicks zu machen oder einen Metreurynter einzulegen versucht hatten, geben doch zu denken.

Von den an Sepsis Verstorbenen wurden 3 nach vorausgegangener Tamponade hochfiebernd von auswärts eingeliefert, 2 weitere waren draussen ebenfalls tamponiert worden und nur eine einzige Frau (1915 A. N. 528) kam.untamponiert und ohne Fieber in die Klinik. Bei ihr musste wegen lebensbedrohlicher Blutung die Totalexstirpation des Uterus gemacht werden. Die durch Schwangerschaftsblutung schon sehr geschwächte Patientin erlag am 19. Tage post operationem einer metastasierenden Allgemeininfektion. 


\section{Mütterliche Morbidität.}

Von den' nach Abzug der 21 Gestorbenen verbleibenden 213 Frauen fieberten im Wochenbett $61=28,6 \mathrm{pCt}$. Davon wiesen Temperatursteigerungen bis $38,5^{\circ} 23$ Frauen auf, während Fieber über $38,5^{\circ}$ in 38 Fällen $=62,2$ pCt. der Fiebernden beobachtet wurde.

Von den 61 fiebernden Frauen waren 29, also nahezu die Hälfte tamponiert.

\section{Kindliche Mortalität.}

Von den 234 Müttern wurden 236 Kinder geboren (einmal Drillinge). Dreimal wurden Missbildungen beobachtet, darunter befindet sich der eine Drilling, der als Foetus papyraceus zur Welt kam. Von den 236 Kindern hatten $150=63,5$ pCt. ein Gewicht über $2000 \mathrm{~g}, 86$ Kinder $=36,5$ pCt. erreichten diesen, in die Placenta praevia-Statistik zur Beurteilung der Lebensfähigkeit allgemein eingeführten Wert nicht. Diese Tatsache, dass rund $1 / 3$ aller Placenta praevia-Kinder schon von vornherein keine oder nur ganz geringe Chancen haben am Leben zu bleiben, muss bei der Wertung der therapeutischen Methoden immer wieder unterstrichen werden.

Von den 236 Kindern kamen 151 lebend und $85=36$ pCt. tot zur Welt. Von diesen waren 30 (darunter 4 mazerierte) schon beim Eintritt der Mutter in die Klinik oder bei Beginn der Operation abgestorben. Zieht man diese 30 schon beim Einsetzen der Therapie. verlorenen Kindesleben von den obenerwähnten $85 \mathrm{ab}$, dann bleiben 55 tote $=23,3$ pCt. Von diesen 55 Kindern hatten 36 ein Gewieht unter 2000 g. Unsere reduzierte kindliche Mortalität beträgt: demnach nur $8 \mathrm{pCt}$.

Von den 151 lebend geborenen Kindern verliessen 93 lebend die Anstalt, 58 wurden zwar lebend geboren, starben aber in den ersten Tagen nach der Geburt: Diese relativ hohe Mortalität der lebend geborenen Placenta praevia-Kinder ist eine schon längst bekannte und gewürdigte Erscheinung. Sčhon Cazeaux schrieb: "Aussi quand il est expulsé vivant, est-il assez souvent grêle et plus faible que dans les conditions ordinaires."

Die Ursache dieser kindlichen Mortalität nach der Geburt ist zum T'eil darin zu suchen, dass die Kinder asphyktisch geboren wurden und dann trotz aller Wiederbelebungsversuche sich doch nicht erholteń, zum Teil ist dem Umstand die Schuld zu geben, 
dass ein Teil der Kinder nicht ausgetragen war und ungeachtet der sorgsamsten Pflege zugrunde ging. Dï

\section{therapentischen Eingriffe}

bei Placenta praevia werden, analog der Einteilung der geburtshilflichen Operation überhaupt, unterschieden in vorbereitende und entbindende Operationen, wozu schliesslich noch die zur Entfernung der Nachgeburt dienende Expression und manuelle Lösung zu rechnen sind.

Nicht in dieses Schema gehört nach unseren gegenwärtigen modernen Ansichten die

\section{Tamponade.}

Bis vor nicht allzu langer Zeit wurde die Tamponade bei Placenta praevia in doppelter Absicht, sowohl zur Blutstillung als auch zur Erregung von Wehen ausgeführt. Speziell der wehenerregende Charakter der Tamponade wurde, vor kurzem noch sehr hoch gewertet. So schrieb noch im Jahre 1893 v. Winckel in seinem Lehrbuch der Geburtshilfe: "Bei der Placenta praevia centralis ist die Tamponade so lange fortzusetzen, bis der Muttermund fast völlig erweitert ist, so dass entweder der vorliegende Kindesteil in ibn eintreten und ihn tamponieren kann oder dass durch Eingehen mit. der Hand und Einleiten des Steisses in den Muttermund eine Tamponade der Plazenta erzielt wird."

Erst die neueste Zeit hat den richtigen Massstab zur kritischen Würdigung dieses "anceps auxilium" (Fr. Hoffmann) gefunden. Am eindrucksvollsten geschah dies auf dem grossen internationalen medizinischen Kongress in London im Jahre 1913, wo im Anschluss an das Referat Döderlein's nahezu sämtliche, aus allen Teilen der Welt zusammengekommenen Geburtshelfer die Tamponade wohl als ganz hervorragendes Mittel zur prophylaktischen Blutstillung anerkannten, ihr aber jede weitere Berechtigung in der Placenta praevia-Therapie absprachen. Der Grund hierfür war die überall gemachte und von allen Seiten bestätigte enorme Infektionsgefährlichkeit der Tamponade.

Auch in unserem Material ist dieser ungünstige Einfluss der Tamponade nachzuweisen. Von unseren 234 Frauen waren insgesamt 107 vor der Geburt tamponiert, davon 24 mehrmals.

Von den 107 tamponierten Müttern erkrankten 29 an Fieber, gestorben sind $11=10,6 \mathrm{pCt}$. 
Diese Mortalität von $10 \mathrm{pCt}$. bedarf nun natürlich insofern. einer Korrektur, als durchaus"nicht alle Todesfälle der Tamponade oder vielmehr einer durch sie veranlassten Sepsis zur Last zu legen sind. Es sind unter den 107 Frauen naturgemäss auch die mitgerechnet, die infolge des starken, ausserhalb der Klinik erlittenen Blutverlustes so geschwächt waren, dass jede Therapie. machtlos blieb und den infausten Ausgang nicht aufhalten konnte. Mit anderen Worten: es finden sich unter'den 10 pCt. sowohl solche Frauen, die wegen, als auch solche, die trotz der Tamponade ad exitum kamen.

Von den 11 nach Tamponade gestorbenen Frauen sind 6 teils unmittelbar, teils einige Stunden nach ihrer Einlieferung in die Klinik an Anämie verstorben, eine 7. erlag am 6. Tage nach der Entbindung einer floriden Lungentuberkulose mit positivem Bazillenbefund. Es bleiben von den 11 also noch 4 Frauen übrig. Von diesen sind 3 an einer bereits intra ritam bakteriologisch fẹstgestellten Streptokokkensepsis zugrunde gegangen, eine 4. ist einer Peritonitis nach extraperitonealem Kaiserschnitt, bei dem die vordere Umschlagsfalte einriss, erlegen. Von den 3 eben erwähnten septisch zugrunde gegangenen Patientinnen wurde die eine mittels vaginalen Kaiserschnittes, Wendung und Extraktion entbunden, bei der zweiten wurde nur die Wendung, und Extraktion gemacht, im 3. Falle erfolgte die Spontangeburt, es musste aber die Plazenta manuell gelöst werden. In allen diesen Fällen wurden also intrauterine Eingriffe gemacht.

Wenn nun auch so gut wie sicher in allen diesen Fällen die Tamponade zum ektogenen Keimimport oder zur endogenen Keimvermehrung geführt hat, so ist doch immerhin noch der Finwand möglich, dass erst durch den operativen Eingriff eine Verschleppung der Bakterien aus der Vagina in die höheren Abschnitte des Geburtskanales erfolgt ist, und dass es ohne intrauterine Manipulationen nicht zur tödlichen Infektion gekommen wäre. Dieser Einwand verliert allerdings dadurch schon an Bedeutung, dass eben in vielen Fällen von Placenta praevia auch nach der Tamponade intrauterine Eingriffe aus einer Indicatio vitalis gemacht werden müssen. Ganz hinfällig wird er aber in unserem 4. Falle. Hier wurde der abdominelle Kaisersehnitt gemacht, es fielen also ausser der Tamponade alle vaginalen Manipulationen weg und das Kind wurde mit Umgehung des infektionsverdächtigen Teiles des Genitalkanales entwickelt. 
Es sind ron 11 tamponiert gewesenen und ad exitum gekommenen Frauen also 4 mit einer an Sicherheit grenzenden Wabrscheinlichkeit an den Folgen der Tamponade zugrunde gegangen. Das bedeutet 36,3 pCt., also mehr als $1 / 3$ der nạch Tamponade überhaupt ad exitum, gekommenen Frauen. Bezogen auf die allgemeine Sterblichkeit machen die an Tamponade géstorbenen Frauen 19 pCt. (von den 21 überhaupt gestorbenen starben 4 an Sepsis).

Also fast ein Fünftel der bei Placenta praevia überhaupt gestorbenen Frauen sind der Tamponade zum Opfer gefallen.

Das Einfachste wäre nun natürlich, die Tamponade ganz aus der Placenta praevia-Therapie $z u$ streichen. Leider ist dies nicht. möglich. Dies wurde auch in London wiederholt von den verschiedensten Seiten betont. So allgemein die Tamponade als. therapeutisches Mittel zur Erregung von Wehen verworfen* wurde, so einstimmig wurde ihr hämostyptischer Wert anerkannt. Speziell zur Behandlung der Blutungen im Privathause und für die Dauer des Transportes der Kranken in eine Anstalt wurde sie als unentbehrlich bezeichnet. Wenn die Tamponade nun auch als notwendiges Uebel in der Placenta praevia-Therapie anerkannt ist, so muss man doch danach streben, die aus ihr erwachsenden Gefahren auf ein möglichst geringes Mass zu beschränken. Dies ist in erster Linie dadurch zu erreichen, dass man den Gebrauch der Tamponade, soweit es ohne Gefährdung der Kranken geschehen kann, einschränkt. Sicher kann durch sparsame Anwendung dieses durchaus nicht einwandfreien Mittels noch manche an Placenta praevia leidende Frau vor schwerem Siechtum oder vor dem Tode bewahrt werden.

Ist man aber gezwungen, zur Tamponade zu greifen, dann muss oberstes Gesetz sein, sie im allerhöchsten Falle nur 6 Stunden liegen zu lassen. Nach Ablauf dieser Zeit muss sie unbedingt entfernt und, wenn nötig, wieder erneuert werden [Weber ${ }^{1}$ ), Zweifel].

$V$ on den eigentlichen Behandlungsmethoden der Placenta praevia ist die einfachste und ungefährlichste die

\section{Blasensprengung.}

Leider genügt sie nur in einem Teil der Fälle zur Bekämpfung der aus der Placenta praevia resultierenden Gefahren.

1) Verhandl. d. Deutschen Ges. f. Gyn. 1913. S. 506. 
In unserem Material finden sich 29 Fälle von künstlichem Blasensprung, d. h. also nur in etwas mehr als in einem Zehntel aller Fälle $(12,3$ pCt.) kam die Blasensprengung in Betracht. Fast. immer (in 26 Fällen) verlief danach die Geburt spontan, nur in 3 Fällen musste das Kind wegen Schlechtwerdens der Herztöne mit der Zange entwickelt werden.

Da die künstliche Blasensprengung bei Placenta praevia wohl in der überwiegenden Mehrzahl der Fälle vor der völligen Erweiterung des Muttermundes vorgenommen wird, so teilt sie die Nachteile des spontanen frühzeitigen Blasensprungs. Solches sind einerseits die 'abnorme Verzögerung und Erschwerung der Geburt durch Wegfall der dynamischen Wirkung der Fruchtblase, andererseits die Möglichkeit des Auftretens von Komplikationen, vor allem von Vorfall der kleinen Teile oder der Nabelschnur.

Eine abnorme Geburtsverlängerung infolge des künstlichen Blasensprungs konnten wir in unseren Fällen nicht beobachten. Die durchschnittliche Geburtsdauer betrug bei den Erstgebärenden 19 Stunden und 15 Minuten, bei den Mehrgebärenden 12 Stunden und 21 Minuten. Zum Teil ist dieser rasche Geburtsverlauf sicher auf die nicht in allen, aber in den meisten Fällen angewendeten Pituitrininjektionen zurückzuführen.

Vorfall kleiner Teile sahen wir nie, dagegen fiel einmal die Nabelschnur vor. Es gelang jedoch mittels der Zange ein lebendes Kind zu entwickeln.

Der künstliche Blasensprung ist die Antizipierung eines im weiteren Verlaufe der Geburt eintretenden physiologischen Ereignisses. Er ist von allen bei der Placenta praevia in Betracht kommenden Eingriffen der qualitativ harmloseste und infolgedessen auch prognostisch günstigste. Dies äussert sich auch in der geringen mütterlichen Morbidität und Mortalität.

Wir verloren in unseren 29 Fällen eine einzige Mutter $(3,4$ pCt. Mortalität). Dieser Fall kann jedoch der Klinik nicht zur Last gelegt werden.

Es handelte sich um eine 37jährige Fünftgebärende (1908. G. N: 1023), die in schwer anämischem Zustande in die Klinik eingeliefert wurde. In „halb verblutetem Zustande" war sie ausserhalb der Klinik von einem Arzt nach Blasensprengung ,rasch“ tamponiert worden. Einige Stunden nach Einlieferung in die Klinik erfolgte, ohne dass inzwischen eine neue Blutung aufgetreten wäre, die Spontangeburt eines toten, reifen Knaben. Kurze Zeit nach der Geburt des Kindes trat ganz plötzlich eine sehr starke profuse Blatung auf. - Da der Credé'sche Handgriff nicht žum Ziele führte, wurde zur manuellen Plazentarlösung 
geschritten. Gleich bei dem Eingehen in die Scheide wurde im hinteren Gewölbe ein $3 \mathrm{~cm}$ breiter, quer verlaufender Riss festgestellt, durch den man in den Douglas'schen Raum gelangte. Die Lösung der Plazenta gelang nur sehr schwer und stückweise. Nach ihrer Entfernung wurden Uterus und Vagina sorg̈fältig austamponiert, doch kam Patientin einige Stunden später zum Exitus. Ueber das Zustandekommen des Risses, der ausserhalb der Klinik, wahrscheinlich bei der Tamponade, erfolgte, komnte nichts in Erfahrung gebracht werden.

Fieber hatten drei Frauen $=10,7$ pCt. Bei allen dreien handelte es sich nur um kurz dauernde Temperaturbewegungen, die in spätestens acht Tagen ohne jegliche Komplikationen abklangen.

Von den 29 Kindern kamen 6 tot zur Welt $=20,7$ pCt. Darunter waren zwei mazerierte dritten Grades und zwei waren schon beim Eintritt der Mutter in die Klinik abgestorben, es sind in Wirklichkeit also nur zwei Kinder in Rechnung zu setzen, das würde 6,8 pCt. kindliche Mortalität bedeuten. Dieses Verhältnis gestaltet sich noch günstiger, es erniedrigt sich auf 3,7 pCt. und erreicht den "normalen" Kinderverlust infolge von Weichteilschwierigkeiten, wenn man von den zwei gestorbenen Kindern eines mit einem Gewicht von unter $2000 \mathrm{~g}$ abzieht.

Trotz ihrer guten Prognose für Mutter und Kind wird die künstliche Blasensprengung nicht immer die auf sie gesetzten Hoffnungen erfüllen.

Die Gründe hierfür sind verschiedene; einmal können die bereits oben erwähnten Komplikationen infolge des frühzeitigen Fruchtwasserabflusses eintreten. Weiter wurde häufig beobachtet - wenn auch allerdings nicht in unseren Fällen - dass die Blutungen doch nicht standen. Dies war besonders dann der Fall, wenn die Blasensprengung ganz im Beginne der Geburt, bei noch kaum eröffnetem Muttermunde, vorgenommen wurde. Endlich kommt es häufig späterhin noch zu sekundären Lageveräriderungen des Kindes, die zu weiteren operativen Eingriffen zwingen. Kurz, es können sich aus dem einfachen Fruchtwasserabfluss eine ganze Reihe von recht unangenehmen Komplikationen ergeben.

In jedem auch ganz glatt durch Spontanausstossung des Kindes beendigten Fall von künstlicher Blasensprengung drohen der Mutter in der Nachgeburtsperiode die gleichen Gefahren wie jeder anderen Placenta praevia-Kranken überhaupt.

So kam in unseren 29 Fällen die Plazenta nur 13 mal spontan, 2 mal auf leichten Druck, 9 mal erst auf Credé und 5 mal 
musste sie manuell gelöst werden, weil es infolge der heterotopen Insertion zu mehr oder minder stàrker Adhärenz gekommen war.

Aus allen diesen Gründen ist es verständlich, dass auch die künstliche Blasensprengung eine gewisse Quote von Versagern hat. Dies dokumentiert sich auch in der grossen internationalen Statistik Döderlein's, der aus 309 Fällen von Blasensprengung 0,9 pCt. mütterliche und $25 \mathrm{pCt}$. kindliche Mortalitắt berechnete.

Wenn wir uns nun zu den aktiven Methoden der Placenta praevia-Behandlung wenden, so kommt zunächst als Methode der Wahl die

\section{Wendung nach Braxton Hicks}

in Betracht.

Diese wurde 31 mal gemacht.

8 mal handelte es sich um Placenta praevia totalis, in den übrigen Fällen um Placenta praevia partialis. Nach Vollendung der Wendung wurde in allen Fällen am Fusse des Kindes ein leichter Gewichtszug angebracht. Die Ausstossung des Kindes wurde darnach stets der Natur überlassen. In 10 Fällen mussten im weiteren Verlaufe der Geburt die Arme gelöst und der Kopf mittels des Veit-Smellie'schen Handgriffes entwickelt wẻrden. In $7 `$ Fällen wurde zur Schonung des rigiden Muttermundssaumes bei totem Kind der nachfolgende Kopf perforiert.

Pituitrin wurde früher nach Ausführung der Wendung - nach Braxton Hicks immer gegeben, später sind wir auf Grund eines sehr traurigen Falles, der allerdings nicht dem Pituitrin zur Last fällt, davon abgekommen.

Es handelte sich um eine 38jährige Viergebärende (1914. A. N. 2522) am Ende der Schwangerschaft, die nach Angabe des Mannes draussen schon sehr stark geblutet hatte. Der Mnttermund war zweimarkstückgross, neben der vorliegenden Plazenta waren`Eihäute zu fühlen. Wendung nach Braxton' Hicks, Gewichtszug von $2 \mathrm{~kg}$. Eine halbe Stunde später war der Rumpf bis zu den. Schulterblättern geboren. Die Entwicklung des Kopfes und der Arme gelang ganz leicht und glatt. Die Plazenta musste manuell gelöst werden. Auch nach ihrer Entfernung blutete es heftig weiter, doch stand die Blutung schliesslich auf kräftige Tamponade hin. Trotzdem verfiel die Patientin immer mehr und war 4 Stunden post partum eine Leiche. Das Ganze machte den typischen Eindruck eines jener Fälle von Placenta praevia, die schon schwer ausgeblutet in die Klinik kommen und hier dann den bei der Geburt unvermeidlichen Blutverlust nicht mehr zu ertragen vermögen. Die Autopsie ergab aber einen mehrere Zentimeter langen Riss im unteren Uterinsegment. Von hier aus war eine starke Blutung in das rechte Parametrium und in das retroperitoneale Bindegewebe erfolgt.' 
Wenn nun hier auch natürlich der Einwand möglich ist, dass der Zervixriss schon bei Ausführung der Wendung erfolgte, so halten' wir es doch für im höchsten Grade wahrscheinlich, daśs der Isthmus uteri erst beim Durchtritte des Kopfes einriss. Irgend ein Wehenmittel war in diesem Falle nicht gegeben worden, und wir halten es auf Grund dieser Beobachtung für besser, nach Ausführung der vorzeitigen inneren Wendung bei Placenta praevia kein Pituitrin zu geben. Wenn man schon die Methode von Braxton Hicks anwendet, dann muss man eben auch ihre Konsequenzen auf sich nehmen, d. h. man muss den Mut haben, im Interesse der Mutter das. Kind absterben zu lassen. Unser obiger Fall zeigt, dass die Mutter auch bei diesem, eigens zu ihrer Rettung unternommenen, Eingriff, sich durchaus nicht ausser Gefahr befindet.

Ausser diesem eben erwähnten Todesfall haben wir bei unseren Fällen von Wendung nach Braxton Hicks noch einen weiteren zu beklagen.

Es handelte sich um eine 44jährige Erstgebärende (1908. G. N. 620a), die in schwer ansgeblutetem Zustande mit kaum fühlbarem Puls in die Klinik eingeliefert wurde. Die Kranke gab an, dass sie schon vor 4 Wochen eine sehr starke Blutung gehabt habe. Der herbeigerufene Arzt habe diese aber auf ein "Gewächs" geschoben. Erst bei der vor einigen Stunden wieder aufgetretenen Blutung habe der Arzt die vorliegende Nachgeburt gefühlt. Er habe dann einen Kollegen herbeigeholt und die beiden Aerzte hätten sich 5 Stunden lang um die Patientin bemüht, jedoch ohne Erfolg. Als der Zustand immer bedrohlicher wurde, hätten die Aerzte der Kranken geraten, sich in die Klinik zu begeben. Hier wurde sofort die Wendung nach Braxton Hicks gemacht. Kindliche Herztöne waren - schon bei Ankunft der Patientin nicht mehr zu hören. Nach der Wendung stand die Blutung. Die Kranke erholte sich jedoch nicht mehr, sondern kam einige Stunden nach Ausstossung des Kindes unter den Zeichen der Herzschwäche zum Exitus.

Unsere mütterliche Mortalität beträgt demnach 6,4 pCt.

Die Nachgeburt kam 6 mal spontan, doch musste in einem dieser Fälle ein fünfmarkstückgrosser Plazentarrest digital entfernt werden, 7 mal kam die Plazenta auf leichten Druck, 2 mal erst auf Credé und 15 mal musste sie manuell gelöst werden.

Das Wochenbett verlief, wenn wir die beiden Todesfälle abziehen, in 24 Fällen völlig fieberfrei. 5 Frauen fieberten, darunter nur 2. schwer, doch verliessen auch diese nach spätestens drei Wochen geheilt die Klinik. Von den 5 Fiebernden waren 2 ausserhalb der Klinik wiederholt tamponiert worden, und gerade bei 
diesen beiden musste die Plazenta manuell gelöst werden. Bei der dritten Frau war, ebenfalls ausserhalb der Klinik, die Blase gesprengt worden, die vierte und fünfte waren nicht tamponiert, die Plazenta kam bei ihnen spontan.

Die kindliche Mortalität betrug in unseren 31 Fällen von vorzeitiger innerer Wendung $21=67,7 \mathrm{pCt}$. Sie stimmt mit den übrigen, in der Literatur niedergelegten Zahlen überein. Allerdings müssen von den 21 toten Kindern 4 abgerechnet werden, nämlich ein mazeriertes und 3 schon beim Eintritt in die Klinik abgestorbene, so dass sich die kindliche Mortalität auf 54,8 pCt. reduziert.

Unter den 31 Kindern wogen 11 weniger als 2000 g. Von den 10 lebend geborenen Kindern sind später noch 5 gestorben und 5 haben die Klinik lebend verlassen.

Seinen Standpunkt bezüglich der

\section{Metreuryse}

hat Döderlein in seinem eingehenden Referat in London dahin präzisiert: "Als einen grossen Nachteil der Metreuryse sehe ich von vornherein die Verwendung eines Fremdkörpers an. Es trifft auch hier, was ich schon über die Vaginaltamponade gesagt habe, die Gefahr zu, dass auch bei Einhaltung aller Antisepsis dieser Fremdkörper sich mit Keimen belädt und somit eine Infektionsquelle wird, die wir gerade hier an der Plazentarstelle besonders fürchten. Auch hier wird eine zeitliche Begrenzung der Anwendung oberster Grundsatz bleiben müssen, was aber mit der Durchführung des ganzen Verfahrens nicht immer leicht vereint werden kann.

Ein weiterer, vielfach beklagter Nachteil der Metreuryse ist, dass die Einführung des Ballons in das Eiinnere, namentlich, wenn man dazu die Durchbohrung der Plazenta nötig hat," keineswegs ein technisch so einfacher Eingriff ist, dass er den Praktikern, für die diese Methode besonders in Betracht kommt, empfohlen werden kann. Man vergegenwärtige sich nur, welch furchtbare Blutungen gelegentlich durch einfaches Berühren der Plazenta beim Touchieren auftreten und wie dann durch diesen Blutstrom das ganze Operationsgebiet, das bei der Einführung des Metreurynters doch dem Auge zugänglich gemacht werden muss, überschwemmt wird. Gelingt es bei lateralem Sitz der Plazenta neben ihr Eihäute aufzufinden und durch sie den Metreurynter einzu- 
führen, dann dürfte diese Gefahr natürlich nieht zu fürchten sein, und hier dürfte die Metreuryse am ehesten am Platze sein."

Entsprechend diesem Standpunkte Döderlein's ist die Zahl der Metreurysen in unserem Material. nur gering, sie beträgt im ganzen 6 Fälle. Stets wurde der Ballon intraamnial gelegt.

Dreimal erfolgte im Anschluss an die Metreuryse die Spontangeburt, doch trat diese in einem Falle erst $251 / 2$ Stunden nach Ausstossung des Metreurynters ein. Zweimal wurde die Wendung nach Braxton Hicks gemacht, einmal musste das unreife tote Kind perforiert werden.

Die Plazenta kam einmal auf leichten Druck, zweimal auf Credé, dreimal musste sie manuell gelöst werden.

Gestorben ist von den mit Metreuryse behandelten. Frauen keine. Im Wochenbett fieberten 2 Frauen, davon die einẹ fünf, die andere sechs Tàge, bei den übrigen verlief das Wochenbett vollkommen fieberfrei.

Von den Kindern leben zwei, drei wurden tot geboren, davon war das eine schon beim Eintritt der Mutter in die Klinik abgestorben, die beiden anderen waren lebensunfähig, das sechste Kind war ebenfalls unreif und starb am 6. Tage nach der Geburt.

Eine gewisse Sonderstellung nimmt unter allen Behandlungsmethoden der Placerita praevia die

\section{Wendung}

oder genauer die "rechtzeitige innere Wendung" (Zangemeister) ein. Wohl alle Geburtshelfer sind sich darüber einig, dass sie für jeden Fall von Placenta praevia, in dem ihre Vorbedingungen gegeben sind, die beste Behandlungs- und Entbindungsmethode darstellt.

Leider ist ihr Anwendungsgebiet nur ein beschränktes. So war sie unter unseren 234 Fällen von Placenta praevia nur $18 \mathrm{mal}$, d. h. also nur in $7,6 \mathrm{pCt}$. aller Fälle möglich. In allen Fällen wurde, eventuell unter Zuhilfenahme kleiner Muttermundsinzisionen unmittelbar an die Wendung die Extraktion des Kindes angeschlossen.

In 10 Fällen handelte es sich um Placenta praevia centralis, in 7 um Placenta praevia lateralis und in einem Falle war die zentral inserierte Plazenta in die Scheide prolabiert.

Als Kindslagen fanden sich 10 Querlagen, darunter 2 mal Nabelschnurvorfall und 1 mal Armvorfall, ferner 7 Schädellagen, 
1 mal bei einer Frühgeburt im 7. Monat fehlt eine Angabe über die Lage.

Von den 18, Müttern sind drei gestorben, das bedeutet also eine Mortalität von 16,6 pCt.

Von den verstorbenen drei Franen war die eine (1910. G. N. 333) eine 22 jährige, in 7. Monat gravide, Erstgebärende. Nachdem sie draussen schon 8 Tage lang bald stärker, bald schwächer geblutet hatte, wurde sie nach einer sehr starken Blutung, wobei sie ohnmächtig geworden war, mit kleinem frequenten Puls und hochgradiger Atemnot in die Klinik eingeliefert. Da der Muttermund fast völlig eröffnet war, wurde sofort die Wendung und Extraktion gemacht. 6 Stunden nach der Operation wurde die Tamponade entfernt. Im Anschluss daran trat ein Kollaps ein, von dem die Patientin sich nicht mehr erholte. Die Autopsie ergab nirgends eine Verletzung und auch keine innere Blutung.

Die zweite Patientin (1912. A. N. 1929). eine 36jährige Achtgebärende hatte draussen in den letzten 4 Tagen ebenfalls stark geblutet. Bei ihrem Eintritt in die Klinik wurde sie, da sie augenblicklich nicht blutete und der Muttermund erst für zwei Finger durchgängig. war, tamponiert. Zwei Stunden später wurde die Tamponade plötzlich durch eine enorm starke Blutung ausgestossen. Es wurde sofort zur Wendung geschritten. Während der Narkose hörten plötzlich die Atmung und Herztätigkeit auf und es gelang trotz aller Bemühungen nicht mehr, die Patientin zum Leben zurïckzurufen.

Im dritten Falle (1916. G. N. 69) handelte es sich um einen mit Placenta praevia kombinierten Abort im 6. Monat. Die Patientin, die draussen von einer Hebamme mit Wattekugeln tamponiert worden war, hatte schon bei ihrer Aufnahme in die Klinik eine Temperatur von $39,0^{\circ}$. Der $30 \mathrm{~cm}$ lange Fötus wurde gewendet und extrahiert. Die Plazenta wurde manuell entfernt. Sie verbreitete schon einen sehr üblen Geruch. Einige Stunden nach dem Eingriff bekam die Kranke einen Schüttelfrost. In den nächsten 48 Stunden verschlimmerte sich unter fortdauernden hohen Temperaturen das Allgemeinbefinden derart, dass zur Laparotomie geschritten wurde. Bei Eröffnung der Bauchhöhle waren Peritoneum und Darmschlingen glatt und glänzend und frei von allen Entzündungserscheinungen. Das rechte Parametrium war verdickt und ödematös. Beim Einschneiden entleerte sich aus den Gewebsspalten dünnflïssiger Eiter. Die durchtrennten Gefässe zeigten eine starre infiltrierte Wand, aus ihrem Lumen ragten vereinzelte Thromben hervor. Nach Exstirpation des puerperalen Uterus samt den Adnexen wurde die rechte Hypogastrika frei präpariert und direkt unterhalb ihres Abganges unterbunden. Am Tage nach der Operation trat noch ein Sehüttelfrost auf, dann fiel das Fieber ab und auch das subjektive Allgemeinbefinden besserte sich ganz auffallend. Zwei Tage später trat aber unter Schiittelfrost ein neuer Temperaturanstieg ein und am 12. Tage nach der Operation erfolgte unter den Zeichen der Herzschwäche der Tod. Aus dem während der Operation und 5 Tage später entnommenen Blute wurden anaerobe Keime gezüchtet.

Von den nach Abzug der drei verstorbenen übrigbleibenden 15 Müttern fieberten vier, also 26,6 pCt., darunter eine leicht und drei schwerer. 
Von den 18 Kindern kamen 6 lebend und $12=66,6, \mathrm{pCt}$. tot zur Welt. Eines von den 12 Kindern war allerdings schon beim Eintritt der Mutter in die Klinik abgestorben, so dass sich unsere kindliche Mortalität auf $61,1 \mathrm{pCt}$. erniedrigt. Von den 12 toten Kindern waren nun allerdings 3 reif und $\mathrm{zu}$ diesen gehörte auch das eben erwähnte, schon beim 'Eintritt in die Klinik abgestorbene. Von den 6 lebenden Kindern waren 2 unter $2000 \mathrm{~g}$ und es gelang trotz aller Mühe und Sorgfalt nicht, sie am Leben zu erhalten.

Wenn wir uns nun dem

\section{vaginalen Kaiserschnitt}

zuwenden, so muss zunächst betont werden, dass er die Operation der Wahl bei den meisten unserer Placenta praevia-Fälle war.

Stets wurde die Hysterotomia vaginalis anterior in der von Döderlein angegebenen Modifikation gemacht.

Es liegt ja zunächst der Gedanke nahe, in der Ausführung des vaginalen Kaiserschnitts bei Placenta praevia zu individualisieren, derart, dass man bei der Insertion der Plazenta an der Vorderwand des Isthmus die Hysterotomia vaginalis posterior macht, während man die vordere Hysterostomatomie nur für die Fälle reserviert, in denen sich die Placenta praevia hauptsächlich an der Hinterwand des unteren Uterusabschnittes befindet. In der Tat 'hat nun auch Döderlein ${ }^{1}$ ) während seiner Tübinger Zeit 12 Fälle von Placenta praevia mit Hysterotomia posterior und nachfolgender Wendung und Extraktion behandelt. Von den 12 Operierten sind 4 ad exitum gekommen, und zwar starben żwei 3 und 4 Tage nach der Operation an septischer Peritonitis, die beiden anderen gleich nach der Geburt an Verblutung.

Ausschlaggebend für die klinische Würdigung der Hysterotomia posterior waren für Döderlein die beiden Fälle von. Peritonitis. Bei der Verlegung des Schnittes in die hintere Zervixwand muss, wenn anders man bis zum inneren Muttermund gelangen will, und dies ist zur Vermeidung von Rissen unbedingt erforderlich, nach Lage der anatomischen Verhältnisse unter allen Umständen die Bauchhöhle eröffnet werden. Dieses unvermeidliche Ereignis ist nun gerade bei den Placenta praevia-Fällen besonders zu fürchten, da diese ja in der Regel untersucht oder tamponiert, also häufig

1) Arch. f. Gyn. Bd. 92. S. 92. 
infiziert, in die Klinik kommen. Wird nun die Bauchhöhle eröffnet, dann ist eine Einwanderung der Infektionserreger aus der Scheide nicht zu verhindern und der Ausbruch einer tödlichen Peritonitis die nur allzu oft eintretende Folge.

Diese Gefahr der Komplikationen von seiten des Peritoneums ist nun bei der vorderen Hysterotomie um ein Vielfaches geringer. Der Grund hierfür liegt in der Syntopie der Excavatio vesicouterina and darin, dass sich die mit ihrer Unterlage, speziell mit der Vorderfäche des ${ }^{\prime}$ Uterus, nur locker verbundene vordere Umschlagsfalte bis in die Gegend des inneren Muttermundes hinauf abschieben lässt. Infolgedessen gehört hier eine Eröffnung der Bauchhöhle zu den Seltenheiten. - Die vordere und die hintere Hysterotomie zeigen infolgedessen die gleiche klinische und prognostische Differenz wie die extra- und intraperitonealen Methoden des abdominellen Kaiserschnittes.

Die Technik des vaginalen Kaiserschnittes gestaltet sich nach dem von Döderlein schon wiederholt angegebenen Vorgehen folgendermassen: Nach Einstellen der Portio mit zwei breiten Plattenspekulis wird die vordere Muttermundslippe mit zwei Winterschen Fasszangen gefasst und vorsichtig nach abwärts gezogen. Nun wird mit der Schere ein ca. $1 \mathrm{~cm}$ langer Schnitt in die vordere Muttermundslippe gemacht. Das eine Scherenblatt wird dann dicht unter der Schleimhaut der Scheide vorgeschoben und die vordere Scheidenwand wird etwa bis zur Mitte ihrer Länge median gespalten. Nach unseren Eriahrungen ist darauf zu achten, dass dieser Schnitt in die Scheidepschleimhaut nicht zu kurz ausfällt, da sonst die Uebersicht über das Operationsfeld erschwert wird und die Blase entschieden nicht, so beweglich und leicht abschiebbar ist. Nun wird der freiliegende Teil der vorderen Zervixwand mit Klemmen gefasst und nach abwärts gezogen. Schon das Tiefziehen des Uterus bewirkt häufig ein gans automatisches Zurückweichen der Blase nach oben. Durch weiteres stumpfes Abschieben der Blase gelingt es nach und nach, immer grössere Teile der vorderen Zervixwand freizulegen. Diese wird unter Einsetzen neuer Klammern hoch hinauf gespalten. Die sich in das Wundgebiet hereindrängende Harnblase wird zusammen mit der vorderen Scheidenwand durch ein Plattenspekulum zurückgehalten. Dije eingesetzten Klammern bleiben liegen und es werden immer neue häher eingesetzt, bis der Schnitt in der vorderen Uteruswand je nach den Umständen eine Länge von $7-10 \mathrm{~cm}$ erreicht hat, $\mathrm{S} \theta$. dass die 
Wendung und Extraktion des Kindes leicht und ohne die Gefahr des Weiterreissens ausgeführ werden können. Auf die Plazenta wird zunächst gar nicht geachtet, auch auf ihren Sitz wird keine Rücksicht genommen. Bei einiger Uebung gelingt es leicht, innerhalb 1-2 Minuten von Beginn der Operation an gerechnet, den Uterus so weit aufzuschneiden, dass die Entbindung nunmehr unmittelbar erfolgen kann. Wir sahen aber auch schon oft dieses Ziel in Bruchteilen einer Minute erreicht. Nun werden die Klammern und Spekula rasch entfernt, die Hand dringt in das Eiinnere vor, wendet das Kind und extrahiert es. Alsdann wird die Plazenta, die ja sowieso schon meistens zum grössten Teil gelost ist, manuell entfernt, die Zervix wird wieder angehakt und der Uterus mit Jodoformgaze fest austamponiert1). Zwischen diesen verschiedenen Phasen der Operation darf nicht die kleinste Pause eintreten. Die einzelnen Stadien müssen sich zeitlich eng und unmittelbar aneinander anschliessen. Nach Vollendung der Tamponade werden die Wundränder wieder genau so wie zu Anfang der Operation mit Klammern gefasst, der Schnitt wird bis zum oberen Ende eingestellt und die vordere Uteruswand wird zunächst für sich durch eine fortlaufende Katgutnaht vereinigt. Dann wird die vordere Scheidenwand ebenfalls. durch eine fortlaufende Katgutnaht auf die Zervixwand aufgenäht. Hierbei ist darauf zu achten, dass zwischen ihr und ihrer Unterlage, also Blase und Uterus, keine toten Räume entstehen. - Wenn je in der Geburtshilfe, so ist hier schnelles Operieren ausschlaggebend

1) Wärend der Extraktion injizieren wir $1 \mathrm{ccm}$ Coluitrin oder Hypophysin intravenös. Die im Durchschnitt nach einer Minute eintretenden kräftigen Wehen erleichtern die Ablösung der Plazenta und wirken entschieden auch günstig auf Atonien. Wenn auch die Muskulatur des Isthmus gegenüber der Muskulatur des Korpus relativ schwach ist, so wäre es doch, wie man sich in vielen Fällen, speziell von Placenta praevia, überzeugen kann, falsch, wenn man ibr jeden Einfluss auf die Blutstillung überhaupt absprechen wollte. Dass bei einer beträchtlichen chorialen Invasion, wie sie von Pankow, uns u. a. gefunden wurde, der Blutstillungsmechanismus mehr oder minder versagen kann, liegt auf der Hand. In solchen Fällen kann ja aber auch jede andere Blutstillungsmethode versagen und die Totalexstirpation des Uterus nötig werden. Jedenfalls möchten wir an diemstare darauf hinweisen, dass die von Sachs (Monatsschr. f. Geb. u. Gy $\$ B(3 \times 0$, S. 544) in die Geburtshilfo eingefübrten intravenösén Pituitroujegrionon sieh uns ganz ausgezeichnet bowälrt haben, und dass wir sto, speziell auch zur Bekämpfung von Nachgøburtsblutungen, nicht mabr missen möchten. 
für den Erfolg. „Die Operation muss so schnell gehen, dass die Kranke gar keine Zeit hat, sich zu verbluten." (Döderlein.)

Mit dieser Methode wurden nun in den letzten 10 Jahren 125 Fälle von Placenta praevia behandelt. Dies dürfte wobl die grösste Zahl von vaginalen Kaiserschnitten bei Placenta praevià sein, die bis jetzt überhaupt von einem Operateur erreicht worden ist.

Von 125 Frauen sind insgesamt, ohne allen Abzug, $12=$ 9,6 pCt. ad exitum gekommen.

Diese Zahl hat nun verschiedene Qualitäten. Zunächst ist sie niedrig im Vergleich zu den früheren Resultaten des vaginalen Kaiserschnittes bei Placenta praevia. Döderlein berechnete nämlich seinerzeit in London im Jahre 1913 aus verschiedenen klinischen Statistiken eine mütterliche Mortalität von $11,3 \mathrm{pCt}$. Unsere Resultate. 'sind also im Vergleich mit dieser Statistik entschieden als gute zu bezeichnen. Sie sind andererseits aber anscheinend durchaus nicht besser als die durchschnittlichen Resultate, wie sie auch mit den anderen Methoden der Placenta praeviaBehandlung erzielt werden.

Es ist nun eine eigene Sache mit den Statistiken. Der alte Satz: "La statistique est le mensonge en chiffres" hat eine doppelte Bedeutung. Einerseits können Ziffern einen Schein von Wahrheit vortäuschen, wo keiner vorhanden ist, andererseits ist es. aber auch möglich, dass sich die Wahrheit hinter Ziffern vierbirgt. Gewiss hat Döderlein ${ }^{1}$ ) recht, wenn er meint, "man. sollte die Gesamtresultate mehr berücksichtigen, um dem Subjektivismus des. Autors möglichst wenig Rechte einzuräumen. Wenn man seinen Schlussfolgerungen nur genïgend grosses Material zugrunde legt, dann werden etwaige Sonderfaktoren in der Statistik eine ungefähr gleich hohe Rolle spielen und es erscheint ... richtiger, das gesamte Material ohne jeden Abzug den Schlussfolgerungen zugrunde zu legen, als die' Statistik zu, reinigen', wobei leicht, wenn auch unbeabsichtigt, Täuschungen unterlaufen."

Trotzdem dürfte es aber berechtigt sein, speziell bei der Wertung einer noch relativ neven und jungen Methode, die mit. ihr erzielten. Resultate kritiseh zu sichten.

Hier sind in unserem Material nun zunächst 2 Fälle $z u$ erwähnen, in denen es zur Aufpfropfung einer Placenta praevia auf

1) Arch. f. Gyn. Bd. 92. S. 95. 
einen durch andere Erkrankungen bereits dem Tode preisgegebenen. Organismus gekommen war.

In dem einen dieser Fălle (1911 A. N. 439) handelte es sich um eine 34 jährige Zehntgebärende, die an einer floriden Lungen- und Larynxtuberkulose mit positivem Bazillenbefund litt und am 6. Tage post partum im Anschluss an eine profuse Hämoptoe ad exitum kam.

Der andere Fall (1913 A. N. 83) betraf eine 25jährige Zweitgebärende, die von dem poliklinischen Assistenten in der Wohnung in vollständig ausgeblutetem Zustande angetroffen wurde: Das Bett der Pat. war buchstäblich von Blut durchtränkt. Die Frau war in höchstem Grade anämisch, machte daneben aber doch auch einen merkwürdig kachektischen Eindruck; der Puls war klein, fadenförmig, kaum fühlbar. Nach ausgiebiger Scheidentamponade wurde die Kranke in die Klinik gebracht. Hier wurde alsbald der vaginale Kaiserschnitt gemacht. Hierbei wurde die tief herabgezogene und fest verwachsene Blase in Einpfennigstückgrösse verletzt. Die Wendung und Extraktion eines $38 \mathrm{~cm}$ langen und $2000 \mathrm{~g}$ schweren, schon abgestorbenen Kindes verlief. ebenso wie der ganze Eingriff vollkommen blutleer. Schon während der Operation fiel eine harte Infiltration der hinteren Scheidenwand auf. Die Untersuchung per rectum ergab, dass ein weit fortgeschrittenes, das ganze Rektum zusammenschnürendes, zerfallendes Rektumkarzinom vorhanden war. Die Pat. verschied bald nach der Operation. Leichendiagnose: Ausgedehntes exulzerierendes Rektumkarzinom, Polyposis intestinalis, Karzinommetastasen im Douglas, Endocarditis chronica, Anämie.

Nach Abzug dieser beiden Fälle reduziert sich unsere mütterliche Mortalität auf 7,2 pCt.

Von den übrig bleibenden 10 Frauen sind 3 einer Sepsis erlegen, die übrigen 6 sind an Anämie zugrunde gegangen.

Ueber den einen der Sepsisfälle hat seinerzeit schon Herr Geheimrat Döderlein ${ }^{1}$ ) berichtet. Es handelte sich um eine tamponiert and bereits hochfiebernd eingelieferte Frau (1909 G. N. 584), die im Anschluss an die Entbindung weiter fieberte und in der dritten Woche post partum einer Thrombophlebitis puerperalis erlag, nachdem die Unterbindung der beiden Venae hypogastricae ohne Erfolg vorgenommen worden war.

Die zweite Patientin (1911 A. N. 476) wurde mit einer Tamponade eingeliefert, die schon seit 16 Stunden lag. Kindliche Herztöne wurden bei der Einlieferung in die Klinik nicht gehört. Wegen der Grösse des Kindes musste bei der Hysterostomatomie der Schnitt hoch hinauf in den Uterus geführt werden. Bei der Extraktion riss, trotz Perforation des nachfolgenden Kopfes, die Schnittwunde weiter, wabei das Peritoneum eröffnet wurde. Die Plica vesico-uterina wurde sofort wieder geschlossen, dann wurden Uterus und Scheidenwand vernäht. Das Kind war mazeriert I. Grades. Schon am Abend des ersten Operationstages stieg die .Temperatur auf $40,1^{\circ}$. Das Fieber hielt sich in den folgenden Tagen auf ungefähr dieser. Höhe und am 14. Tage erfolgte unter den Zeichen

1) Arch. f. Gyn. Bd. 92 . S. 94. 
der allgemeinen Sepsis der Tod. - Die Leichendiagnose lautete: Sepsis, jauchige Parametritis und Endometritis, parencliymatöse Degeneration des Herzmuskels. Verrukôse Endokardwucherungen auf den Mitralund Aortenklappen, beginnende desquamative Pneumonie der rechten Lunge, starkes Lungenödem, septische Milzschwellung, Oedem und parenchymatöse Degeneration der Nieren.

Im dritten Falle handelte es sich um eine 33jänrige Sechstgebärende (1915 A. N. 528), die in sehr anämischem Zustande eingeliefert wurde. Pat. gab an, seit mehreren Wochen beständig zu bluten. Der vor 5 Tagen wegen starker Blutung konsultierte Arzt hatte der Pat. geraten, zunächst abzuwarten, bis die Blutung sistiere und sich dann in die Klinik zu begeben. - Typischer vaginaler Kaiserschnitt. Nach Entleerung des Uterus sehr heftige Blutung aus dem unteren Uterinsegment, die auch auf Tamponade nicht stand. Es wurde deshalb die Totalexstirpation des Uterus vorgenommen. Am 19. Tage post operationem Exitus unter den Zeichen der Sepsis.

Es mögen nun noch kurz die Krankengeschichten der an Verblutung gestorbenen Frauen folgen.

1. 27 jährige Erstgebärende (1911 G. N. 850) wird in sehr anämischem Zustande untamponiert in die Klinik eingeliefert. Der Ehemann gab an, dass die Pat. draussen schon "etwa 3 Liter Blut" verloren habe. Vaginaler Kaiserschnitt, Wendung und Extrakțion. Kind $45 \mathrm{~cm}$ lang und $2300 \mathrm{~g}$ schwer, lebt. Bei der Operation mässige Blatung, ca. $300 \mathrm{~g}$. Bald danach nimmt der Puls an Kraft zusehends ab, die Pat. wird unruhig, fängt heftig an zu husten. Bei dieser Gelegenheit wird ein Teil der Scheidentamponade herausgepresst. Hierbei fliessen nur $30 \mathrm{ccm}$ Blut ab. Sofortige Nachtamponade. Die Herzkraft sinkt jedoch. immer mehr und 4 Stunden post operationem erfolgt der Exitus.

2. 32 jährige Zweitgebärende (1910 S. A. 52). Pat. hatte schon Wochen vorher geblutet, darunter in den letzten 8 Tagen sehr stark. Nach einer sehr schweren Blutung (ca. 1 l) kam die Pat. sehr anämisch in die Klinik. Vaginaler Kaiserschnitt. Schon bei Beginn der Narkose Kollaps. Bei der Operation war die Blutung nur sehr gering. Plazenta. auf leichten Druck. Nach der Operation erholte sich die Pat. sehr gut. Einige Stunden später -wurde der bis dahin steinharte Uterus plötzlich weich, Blut sickerte durch die Tamponade hindurch und trotz aller Gegenmassregeln trat bald darnach der Tod ein.

3. 23jährige Zweitgebärende (1912 A. N. 885) wurde schwer ausgeblutet in die Klinik aufgenommen. Vaginaler Kaiserschnitt. Blutverlust bei der Operatión mässig. Eine Stunde nach der Operation war die ganze Tamponade durchblutet und unter dem Zeichen der Anämie erfolgte der Exitus.

4. 37 jährige Zehntgebärende (1913 A. N. 1970). Die Pat. war schon im. Oktober 1913 wegen Schwangerschaftsblutungen in der Klinik gewesen, war aber nach einigen Tagen entgegen dringendem ärztlichem Rat auf eigene Verantwortung wieder ausgetreten. Am 9. Xl. kam sie in sehr anämischem Zustande mit kleinem, weichem, frequentem Puls wieder in die Klinik. Der Muttermund war für einen Finger durchgängig, in ihm fühlte man ringsum Plazentargewebe. Vaginaler Kaiserschnitt. Das reife, lebende Kind wurde durch Wendung und Extraktion entwickelt. Nach der Operation wurde trotz sorgfältiger Tamponade 
der Uterus nicht recht hart, weshalb die Gauss'sche Aderpresse angelegt wurde. 'Etwa $1 / 2$ Stunde später klagte die Frau über heftige Parästhesien in den Beinen, der Puls hatte sich bedeutend gebessert. Es wurde deshalb die Aderpresse abgenommen, doch war dies sofort von einem schweren, ohnmachtähnlichen Kollaps und einer neuen Blutung gefolgt. Die Presse wurde deshalb von neuem angelegt. Auf eine ernente Lösung einige Zeit später folgte eine erneute Blutung. Da die Frau immer weiter blutete, wurde die Totalexstirpation des Uterus gemacht, doch überstand die schwer ausgeblutete Pat. diesen Eingriff nicht, sondern kam bald danach zum Exitus.

5. 36 jährige Neuntgebärende (1914 A. N. 2769). Die Kranke wird "sehr ausgeblutet" aus der Poliklinik eingeliefert. Muttermund für zwei Finger durchgängig, überall von Plazentargewebe bedeckt. Vaginaler Kaiserschnitt; hierbei nur sehr mässige Blutung. Anlegung der Aderpresse. Diese wurde nach einer Stunde entfernt. Einige Zeit später war die Tamponade durchblutet und $2 \frac{1}{2}$ Stunden nach der Operation trat der Tod ein.

6. 30 jährige Zweitgebärende (1915 A. N. 576). Die Kranke wurde in äusserst anämischem Zustande eingeliefert. Die Haut war wachsgelb, der Puls klein, frequent, kaum zu fühlen, die Lippen waren fast weiss. Kindliche Herztöne waren nicht $z \mathfrak{n}$ hören. Vaginaler Kaiserschnitt, Wendung, Extraktion, Perforation des nachfolgenden Kopfes. Manuelle Plazentarlösung. Eisenchloridtamponade des Uterus. Es blutete danach " fast gar nicht" mehr und die Frau erholte sich etwas, bald verschlechterte sich der Puls jedoch wieder, es trat Atemnot anf und 5 Stunden post operationem erfolgte der Exitus.

7. 44 jährige Dreizehntgebärende (1917 G. N. 1907) wurde nach sehr schwerer Blutung von der Hebamme tamponiert in die Klinik eingeliefert. Der Muttermund war nur für einen Finger durchgängig und ringsum von Plazentargewebe bedeckt. Vaginaler Kaiserschnitt. Blutung gering. Einige Zeit nach der Operation verfiel die Frau und kam 21 Stunden nach der Entbindung unter dem Zeichen der Herzschwäche zum Exitus.

Bei allen unseren sieben, an Verblutung gestorbenen Patientinnen handelte es sich also um Frauen, die in schwer ausgeblutetem Zustande in die Klinik eingeliefert wurden. Bei allen lag Placenta praevia centralis vor. Mit Ausnahme von einer, die erst in der zweiten Hälfte des 10. Monats zu bluten angefangen hatte, waren bei allen schon in früheren Schwangerschaftsmonaten zum Teil sehr starke Blutungen aufgetreten. Unbegreiflicherweise war aber eine Reihe unserer Patientinnen von ibren Aerzten konservativ behandelt worden und kam erst, als die Blutungen einen lebensbedrohlichen Charakter annahmen, in die Klinik. Man wäre versucht, zu behaupten, dass keine Therapie mehr imstande gewesen wäre, diese schwer ausgebluteten Frauen zu retten, wenn es nicht unmöglich wäre, den Beweis für diese Behauptung zu erbringen. 
Jedenfalls wäre es aber leicht gewesen, eine glänzende Statistik des vaginalen Kaiserschnittes zu bekommen, wenn wir diese, schon auf das schwerste geschädigten Fraven nicht mit der vorderen Hysterotomie, sondern mit irgend einer anderen Methode behandelt hätten. Wir sind aber von der Ueberzeugung ausgegangen, dass diesen Frauen nicht nur jed̄̄ mögliche, sondern die beste mögliche Hilfe angedeihen muss. Die beste Methode bei; Placenta praevia ist aber die; welche die Frauen in möglichst. kurzer Zeit und mit dem geringsten Einsatz von Gefahr zu entbinden gestattet. Diesen beiden Anforderungen wird der vaginale Kaiserschñitt gerecht. Wenn wir auch ausserstande sind zu heweisen, dass die sieben Verblutungsfälle unvermeidlich waren und bei jeder anderen Therapie sich auch ereignet hätten, so muss doch betont werden, dass sie nach unserer subjektiven Auffassung dem vaginalen Kaiserschnitt nicht zur Last zu legen sind. Dass der Blutverlust bei der Operation selbst nur indirekt als auslösende Ursache für den infausten Ausgang in Betracht kommt, geht daraus hervor, dass in 6 Fällen der Blutverlust bei der Entbindung unter $300 \mathrm{~g}$ und nur in einem Falle $400 \mathrm{~g}$ betrug. Derartige geringe Blutverluste sind aber bei jeder. Methode unvermeidlich und wären selbst beim Zuwarten und bei völlig spontanem Verlauf der Geburt nicht zu vermeiden gewesen, „denn, wenn dieFrauen so weit entkräftet sind, dass sie nicht mehr $400 \mathrm{~g}$ Blutverlust vertragen, ist jedes Entbindungsverfahren mit der Gefahr. eines unglücklichen Ausganges verknüpft" ( $\mathrm{Z}$ weifel).

Wollte man den vaginalen Kaiserschnitt deshalb verurteilen, so wäre es genau das Gleiche, wie wenn man die Laparotomie bei Extrauteringravidität aufgeben würde, weil nach ihr doch noch. Frauen sterben können. Ebenso wie hier ein gewisser Prozentsatz. von Kranken nicht mehr zu retten ist, ebenso werden bei der Placenta praevia eine gewisse Anzahl von Frauen nicht wegen, sondern trotz des vaginalen Kaiserschnittes ihr Leben verlieren. Dies ist aber nicht der Methode, sondern Zufälligkeiten und Umständen zur Last zu legen, die ausserhalb des Bereiches unserer Macht liegen.

Jedenfalls haben wir im Hinblick auf unsere Resultate keinen Grund, den vaginalen Kaiserschnitt als minderwertig im Vergleich mit den übrigen Methoden zu verzeichnen und ihn zu verlassen.

Komplikationen sahen wir bei der Ausführung des vaginalen Kaisersehnitts nur in einer verschwindend geringen Zahl von 
Fällen. Viermal riss der Operationsschnitt weiter, hierbei wurde zweimal die Plica vesico-uterina éröffnet; der eine, bereits erwähnte Fall, endete letal, es war die oben angeführte Frau, die mit einer seit 16 Stunden liegenden Tamponade in die Klinik kam. Die andere Patientin, bei der die Plika eröffnet wurde, bekam Temperatursteigerungen bis zu $39^{\circ}$, die aber am 6 . Tage post partum zur Norm abfielen. In drei Fällen wurde die Blase verletzt. Hièrvon ist zweimal die Blasenwunde glatt und primär geheilt; bei der dritten Frau wurde später eine erfolgreiche Fisteloperation gemacht.

Das Wochenbett verlief bei den nach Abzug der 12 Gestorbenen verbleibenden 113 Frauen in 44 Fällen $=38,9$ pCt. fieberhaft, doch hatten von diesen 44 Frauen nur $25=23,0$ pCt. (auf die 113 Frauen berechnet) Temperaturen über $38,5^{0}$.

Von den 125 Kindern sind 34 tot zur Welt gekommen, das würde also eine kindliche Mortalität von 27,2 pCt. bedeuten. Von diesen 34 Kindern sind nun 15, also nahezu die Hälfte, schon bei Eintritt in die Klinik abgestorben gewesen, es sind demnach also nur 19 Kinder in Rechnung zu setzen. Die eigentliche kindliche Mortalität beträgt demnach nur 15,2 pCt.

Von diesen eben erwähnten 19 Kindern hatten nun 16 ein Gewicht unter $2000 \mathrm{~g}$, so dass der Ausfall an reifen Früchten nur $3=2,4$ pCt. beträgt.

Von den lebend geborenen Kindern sind nun noch eine Reihe, insgesamt 38 , in den ersten Tagen nach der Geburt zugrunde gegangen. 53 Kinder $=42,4$ pCt. aller geborenen, haben die Klinik lebend verlassen.

Dass der Ausfall an Kindern bei vaginalem Kaiserschnitt so relativ gering ist, ist nicht verwunderlich, da ja die Wendung und Extraktion des Kindes, speziell, wenn die Blase noch steht, rasch und spielend vor sich gehen. Trotzdem darf dieser Ausschlag zugunsten der Kinder nicht in den Vordergrund bei der Begründung der Vorzüge des vaginalen Kaiserschnitts gestellt werden. Die lebenden Kinder müssen vielmehr "gewisssermassen als ein Nebenprodukt unserer Therapie ${ }^{u}$ (Döderlein) betrachtet werden, das wir ja ganz gerne entgegennehmen, aber nur dann, wenn es nicht auf Kosten des mütterlichen Wohles erzielt wird.

Wenn wir noch einmal kurz die Vorzüge des vaginalen Kaiserschnittes in der Behandlung der Placenta praevia zusammenfassen, so sind es folgende: Der vaginale Kaiserschnitt 
gibt uns die Möglichkeit, leichter und sicherer als mit allen anderen Behandlungsmethoden die Hauptgefahr der Placenta praevia, die Blutung, zu beherrschen. Die Verblutungsgefahr wird deshalb vermindert, weil die definitive Blutstillang eine absolut sichere und zuverlässige ist. An Stelle der bei den anderen Behandlungsmethoden möglichen unkontrollierbaren, dem Zufall anheimgegebenen Nebenverletzungen, haben wir bei der vorderen Hysterotomie eine einzige, unter Leitung des Auges gemachte, leicht überseh- ủd kontrollierbare Wunde. Die Beschränkung der Verletzung auf die vordere Zervixwand hat nicht nur eine wesentliche Abkürzung der Operationsdaner, sondern auch eine bedeutende. Vereinfachung der Wundverhältnisse und ihrer Beherrsehung zur Folge. Fin weiterer Vorzug der Methode besteht darin, dass ihr Anwendungsgebiet ein sehr ausgedehntes ist. Im grossen und ganzen kann man sagen, dass alle Fälle von Placenta praevia, die einen Eingriff nötig machen, und nicht durch den Blasensprung oder die rechtzeitige innere Wendung erledigt werden können; in gleicher Weise für den vaginalen Kaiserschnitt geeignet sind. Ein Unterschied zwischen den einzelnen Fällen und Möglichkeiten, ob die Plazenta vorne oder hinten sitzt, ob es sich um Placenta praevia totalis oder partialis handelt, braucht nicht gemacht zu werden. Auch die Reife und das Ausgetragensein der Kinder spielen in der Indikationsstellung keine Rolle. Die Blutung selbst war in keinem unserer Fälle als bedeutend zu bezeichnen. Man muss nur den Schnitt gross genug machen, damit er bei der Extraktion der Kinder nicht weiter reisst. Ferner muss man nach der Entleerung des Uterus das Operationsgebiet gut einstellen, um es klar übersehen zu können.

Wenn wir sagten, der vaginale Kaiserschnitt sei die Operation der Wahl in unseren Fällen von Placenta praevia, so heisst das nicht, dass jetzt einfach an Stelle der Wendung nach Braxton Hicks oder der Metreuryse der vaginale Kaiserschnitt getreten sei. Es bedeutet auch nicht, wie man versucht sein könnte zu glauben, etwa ein Accouchement forcé mit modernen Mitteln.

Blasensprengung, vorzeitige innere Wendung und Metreuryse sind Eingriffe, : welche den Zweck haben, in det Eröffnungsperiode die Hauptgefahr der Placenta praevia, die Blutung, zu bekämpfen. Ist die Eröffnungsperiode beendet, dann ist auch ihre Wirksamkeit zu Ende und es müssen, wenn anders nicht die 
Spontangeburt erfolgt, entbindende Operationen an ihre Stelle treten. Bei den Gefahren, welche nun sogar schon bei Spontangeburt einer Placenta praevia-Kranken drohen, ist es eine wohl allgemein durchgeführte Regel, die Eröffnungsperiode nicht künstlich abzukürzen. Durch eine lange Geburtsdauẹ wird aber andererseits, speziell bei den Fällen, die untersucht oder gar tamponiert wurden, die Infektionsgefahr bedeutend erhöht. Weiter ist auch in nicht allzu seltenen Fällen der Blutstillungsmechanismus kein absoluter. Wenn auch nur vielleicht sehr wenig - etwas Blut rieselt doch häufig neben dem tamponierenden Gegenstande, sei es nun der Kindskörper selbst oder ein Metreurynter, aus den Genitalien heraus. Auch nach der Ausstossung des Kindes blatet es häufig etwas -, zwar nicht so viel, dass man sich sofort zum Eingreifen entschliessen müsste, aber auch andererseits nicht so wenig, dass es für die schon mehr, oder weniger ausgeblutete Kranke gleichgültig sein könnte. - Dann kommt das Warten in der Nachgeburtsperiode. Wenn es auch selbstverständlich ist, dass man ununterbrochen am Gebärbett bleibt, so erwägt man häufig doch, ob die Plazenta nicht vielleicht doch noch spontan kommt, oder ob man ihre Entfernung beschleunigen soll. Aber selbst wenn man dies prinzipiell in allen Fällen tut, so ist auch die Entwicklung der Plazenta durch den Credé'schen Handgriff mit einem gewissen Blutverlust verbunden. Gelingt der Credé'sche Haandgriff aber, wie in so vielen Fällen, infolge mehr oder minder hochgradiger Adhärenz der Plazenta an der Isthmuswand nicht, dann muss schliesslich doch noch zur manuellen Plazentarlösung geschritten werden. Bis dahin kann viel kostbares Blut verloren gegangen sein. Ist dann die Plazenta entfernt, dann wird man in der Regel zusehen, $\mathrm{ob}^{\circ}$ es blutet, und erst, wenn die Blutung stärker ist, tamponieren. Auch dabèi kann wertvolle Zeit verstreichen.

Allen diesen Eventualitäten gehen wir mit dem vaginalen Kaiserschnitt aus dem Wege. Wie wir nämlich schon bei der Beschreibung der Operationstechnik erörtert haben, verstehen wir unter vaginalem Kaiserschnitt nicht das einfache Aúfschneiden, Entleeren und Wiederzunähen des Uterus. Wir verstehen vielmehr darunter eine Summe von Verrichtungen; die sich in jedem Fall regelmässig, in gleicher Reihenfolge und zeitlich eng aufeinander folgen, nämlich: Inzision der Zervix bis zum inneren Muttermund, Entwicklung des Kindes, manuelle Lösung der 
Plazenta, Tamponade des Uterus, Naht der Schnittwunden, Tamponade der Scheide. Auf diese Weise beendigen wir Eröffnungs-, Austreibungs- und Nachgeburtsperiode durch einen einzigen, zeitlich eng begrenzten Eingriff und sichern uns zugleich gegen atonische Nachblutungen. Unser Ziel ist also nicht die Abkür.zung der Eröffnungs- oder Austreibungsperiode an und für sich, sondern unser Ziel ist, die Placenta praevia-Kranke möglichst rasch und sicher über die Gesamtgefahren der ganzen Geburt und der ersten Wochenbettsstunden hinwegzubringen.

Bei der Erfüllung dieser Aufgabe ist der vaginale Kaiserschnitt aber nicht Endzweek, sondern nur Mittel zum Zweck. Dies kann in der ganzen Diskussion über den vaginalen Kaiserschnitt bei Placenta praevia nicht oft und nicht kräftig genug unterstrichen werden.

Der vaginalen Hysterotomie ist nun in den letzten Jahren eine starke Konkurrenz in dem

\section{abdominellen Kaiserschnitt}

erwachsen ${ }^{1}$ ). In erster Linie waren für die Einführung des abdominellen Kaiserschnittes in die Placenta praevia-Therapie massgebend die anatomischen Untersuchungen über die Nidation des Eies im Isthmus uteri. Der durch die Ansiedlung des Eies erfolgende anatomische Umbau der Isthmuswand mit der konsekutiven funktionellen Insuffizienz liessen die Verhütung einer allzu starken und allzu lang dauernden Dehnung des unteren Uterinsegmentes als erwünscht erscheinen. Befürchtet wurden einerseits die Zerreissungen des Isthmus beim Durchtritt des Kindes, andererseits die schwer zu stillenden Nachgeburtsblutungen. Beide Gefahren suchte man durch den abdominellen Kaisérschnitt zu umgehen.

Die Döderlein'sche Klinik hat sich dieser Bewegung nur in bedingtem Masse angeschlossen. Die Gründe hierfür sind folgende:

Im Gegensatz zum vaginalen Kaiserschnitt, der für nahezu alle Fälle von Placenta praevia passt, ist die Indikationsstellung des abdominalen Kaiserschnitts eine beschränkte. Alle Autoren,

1) Anmerkung bei der Korrektur: Die interessante und reichhaltige Arbeit von Jaschke über "Die Leistungsfähigkeit der abdominalen Schnittentbindung bei Placenta praevia" (Zentralbl. f. Gyn. 1918. Nr. 10. S. 172) ist erst nach Fertigstellung des Manuskripts erschienen und konnte leider nicht mehr berücksiohtigt werden. 
die den abdominellen Kaiserschnitt bei Placenta praevia befürworten, betonen, dass er möglichst bald ausgeführt werden muss, da eine seiner Hauptaufgaben darin besteht, die im Verlaufe der Geburt eintretende Dehnung des unteren Uterinsegmentes möglichst zu verhindern. Nach Finkner ${ }^{1}$ ) nimmt Pankow bei über fünfmarkstückgrossem Muttermund Abstand von der Operation. Mo $\mathrm{m} \mathrm{m}^{2}$ ) schreibt, dass der Kaiserschnitt nur dann einen idealen Blutstillungsmechanishus herbeizuführen imstande ist, wenn er "vor einer weiteren Dehnung des Isthmus durch den Geburtsakt, also etwa vor der Zeit, wo der Muttermund kleinhandtellergross ist, ausgeführt wira." Alle Fälle also, in denen der Muttermund kleinhandtellergross. und grösşer ist, müssen auf irgend eine andere Weise entbunden werden.

$\mathrm{Zu}$ dieser an unid für sich schon bedeutungsvollen Kontraindikation kommt eine zweite, noch viel wichtigere hinza. Diese besteht in dem Verbot des abdominellen Kaiserschnittes bei infizierten Fällen.

Dieser Forderung ist leicht nachzukommen in den Fällen, in denen die Frauen fiebernd in die Klinik eingeliefert werden. Schwierig wird aber die Entscheidung bei den nicht fiebernden Kreissenden, die draussen schon untersucht und behandelt wurden. Ganz besonders zu fürchten sind hier die Patientinnen, welche tamponiert gewesen sind. Nun hat aber der weitaus grösste Teil der wegen Placenta praevia in die Anstalt überführten Frauen eine solche suspekte Anamnese. Alle diese Frauen müssen als infektionsverdächtig angesehen und vom intraperitonealen Kaiserschnitt ausgeschlossen werden.

Man hat nun für solche Fälle den extraperitonealen Kaiserschnitt vorgeschlagen. Aber auch dagegen lassen sich Einwände erheben. Als ganz besonders schwerwiegend erscheint uns folgende Tatsache: In einem gewissen Prozentsatz von Fällen, der sich allerdings bei zunehmender Technik automatisch reduziert, gelingt es nicht, den rein extraperitoneal angegangenen Fall nach dem beabsichtigten Operationsplan durchzuführen, sei es nun, dass man wegen Blutungen oder wegen Nichtauffindens der Peritonealfalte gezwungen ist, zum intraperitonealen Kaiserschnitt überzugehen, sei es, dass es zur unbeabsichtigten Verletzung des Peritoneums

1) Zentralbl. f. Gyn. 1917. S. 145.

2) Münch. med. Wochenschr. 1916. S. 889. 
beim Aufsuchen und Empordrängen der Peritonealfalte oder bei der Entwicklung des Kindes kommt. Man hat es also nicht in allen Fällen absolut sicher in der Hand, rein extraperitoneal bleiben zu können. Wird nun aber in einem infizierten Falle von Placenta praevia das Peritoneum eröffnet, dann ist der ganze beabsichtigte Zweck des extraperitonealen Vorgehens vereitelt und die aus dem Uterus durch das Operationsgebiet in die Bauchhöhle gelängten Infektionserreger können leicht zu einer tödlichen Peritonitis führen. Einen derartigen Fall èrlebten wir an hiesiger Klinik.

Es handelte sich um eine 36 jährige Erstgebärende (1912. A. N. 2092) am Ende der Schwangerschaft. Die Patientin hatte draussen sehr stark geblutet und war deshalb tamponiert worden. Der Muttermund war für einen Finger durchgängig und rings von Plazentargewebe überlagert. In Anbetracht der bei der alten Erstgebärenden zu erwartenden Weichteilschwierigkeiten wurde der abdominelle Kaiserschnitt gemacht. Bei dem Versuch, die Umschlagsfalte nach oben zu schieben, riss das Peritoneum ein. Es wurde deshalb von dem extraperitonealen Vorgehen Abstand genommen und der intraperitoneale zervikale Kaiserschnitt gemacht. Die Extraktion des $51 \mathrm{~cm}$ langen und $2400 \mathrm{~g}$ schweren lebenden Kindes gelang leicht. Die Plazenta war im Isthmus fest adhärent und konnte nur mit Mühe gelöst werden. Die Uteruswunde wurde nach ihrer Vereinigung vollkommen mit Peritoneum bedeckt und dadurch völlig aus der Bauchhöhle ausgeschaltet. Am 2. Tage nach der Operation trat unter dem Zeichen paralytischen Ileus der Tod ein. Die Autopsie ergab eine beginnende, eitrig-fibrinöse Peritonitis.

Gewiss macht es einen. Unterschied, ob die Bauchhöhle wie beim intraperitonealen Operieren breit und auf längere Zeit geöffnet ist, oder ob es sich um ein kleines Loch handelt, das sich gleich nach seiner Entstehung durch eine einzige Nabt verschliessen lässt. Jmmerhin kann auch schon die passagere Eröffnung der Umschlagsfalte genügen, eine Infektion des Peritoneums mit allen ihren deletären Konsequenzen ermöglichen.

Eine weitere Kontraindikation gegen den abdominellen Kaiserschnitt bei Placenta praevia überhaupt ist nach Döderlein dann gegeben, wenn "die Frau in wesentlich einfacherer. Weise, .... gefahrlos entbunden werden kann".

Neben diesen Kontraindikationen gegen den abdominellen Kaiserschnitt von Seiten der Mutter gibt es auch solche von Seiten des Kindes. Von allen Vertretern des abdominellen Kaiserschnitts in der Placenta praevia-Therapie werden seine glänzenden Resultate für die Kinder betont. So erfreulich und erstrebenswert bei einer so schweren Dystokie neben der Rettung der Mutter 
auch die Erhaltung des Kindes ist, so darf man doch nicht vergessen, dass gerade bei der Placenta praevia die Verhältnisse für die Kinder besonders ungünstig liegen. Ein grosser Teil der Placenta praevia-Kinder ist unreif oder bei Einsetzen der Therapie schon abgestorben. So befanden sich unter unseren 236 Kindern 86 mit einem Gewicht unter $2000 \mathrm{~g}$ und von den 150 anderen waren 16 schon beim Eintritt in die Klinik abgestorben. Auf diese nicht lebensfähigen und schon abgestorbenen Kinder - in unserem Material $102(86+16)=43,2$ pCt, nach Döderlein rund 50 pCt. - kann und braucht bei der Behandlung. der Placenta praevia - Fälle keine Rücksicht genommen zu werden. Hier kann durch zerstückelnde Operationen, ohne also zu starke Dehnung des Uterinsegmentes, die Geburt auf natürlichem Wege beendet werden.

Bei Berücksichtigung aller dieser Konteaindikationen wird sich die Zahl der für den abdominellen Kaiserschuitt geeigneten Fälle von Placenta praevia naturgemäss bedeutend reduzieren: Auf diesen Punkt hat schon Hannes ${ }^{1}$ ) hingewiesen und auch Kehrer ${ }^{2}$ ) betonte, dass er bëi Anlégung der strengen Indikationsstellung jahrelang auf die Gelegenheit zu einem klassischen Kaiserschnitt bei Placenta praevia habe warten müssen. Demgegenüber hat allerdings Pankow betont, dass der Forderung nach einem infektionsfreien Genitale in Freiburg die Hälfte aller Fälle genügte und auch Finkner hatte die gleiche Quote nicht infizierter Fälle.

Dass der abdominelle Kaiserschnitt nach Ausschaltung der erwähnten Kontraindikationen bei der Behandlung der Placenta praevia Gutes zu leisten vermag, ist nicht zu bestreiten. Ob es ihm aber gelingen wird, die führende Stellung in der klinischen Therapie der Placenta praevia zu erringen, lässt sich heute noch nicht entscheiden.

Wir selbst machten ausser dem bereits erwähnten Falle nur noch zweimal den Kaiserschnitt bei Placenta praevia, so dass wir im ganzen über 3 Fälle verfügen. Die Indikation zur Operation gaben in allen 3 Fällen die rigiden Weichteile alter Erstgebärender mit noch erhaltener Portio und geschlossenem oder kaum für einen Finger durch'gängigem Muttermund. Die Operation verlief, mit

1) Zit. nach Finkner.

2) Zentralbl. f. Gyn. 1914. S. 48. 
Ausnahme des aben angeführten Falles, bei dem das Peritoneum einriss, stets rein extraperitoneal. Die Kinder waren durchweg ausgetragen und gross: Zwei hatten ein Gewicht von je $3400 \mathrm{~g}$, eines war $50 \mathrm{~cm}$ lang un $4900 \mathrm{~g}$ schwer. Alle 3 Kinder blieben am Leben und verliessen gesund die Anstalt.

Zur korrelativen Wertung unserer Resultate der Placenta praevia-Behandlung erscheint uns am geeignetsten das Material v. Winckel's. Wir bekommen so zwei grosse, organisch und chronologisch miteirłander zusammenhängende Vergleichsserien, nämlich

1. das Material v. Winckel's (1884 bis 30. Sept. 1907)

2. " " Döderlein's (1. Okt. 1907 bis 1917).

Bei der vergleichenden Betrachtung dieser Serien ergibt sich eine Reihe interessanter Tatsachen.

In den Jahren vonl 1884 bis Sept. 1907 inkl. waren in den Journalen der Münchener Universitäts-Frauenklinik unter $24951 \mathrm{Ge}-$ burten 154 Fälle von Placenta praevia zu finden. Dies entspricht einer Häufigkeit der Placenta praevia-Fälle ron $1: 162$. Dieses Verhältnis ist nun seit 1907 auf 1:119 gestiegen. Somit ergibt sich auch hier wieder die schon eingangs auf anderem Wege gefundene Tatsache, dass in den letaten Jahren nicht nur absolut, sondern vor allem auch relativ viel mehr Frauen mit Placenta praevia die Klinik aufsuchen als früher.

Von den 154 Müttern der Winckel'schen Statistik starben nun $24=15,6 \mathrm{pCt}$. Mortalität. Demgegenüber beträgt die mütterliche Sterblichkeit in dem Döderlein'schen Materiale 21 Tote auf 234 Fälle, also $8,9 \mathrm{pCt}$. Die Döderlein'sche Mortalität ist also nahezu um die Hälfte geringer als die Winckel'sche.

Wenn wir den Gründen dieser auffallenden Differenz nachgehen, so können wir wohl mit Sicherheit behaupten, dass die Ursache an dem Material als solchem nicht liegen kann. Es handelt sich in beiden Serien, der Winckel'schen sowohl als der Döderlein'schen, um ein kontinuierlich im Laufe der Jahre unter den gleichen Bedingungen aus München und der Umgebung der Klinik zugeflossenes Material. Der einzig mögliche Einwand, dass infolge der besseren Beförderungs- und Verkehrsverhältnisse der Neuzeit die Patientinnen in besserer Verfassung in die Klinik eingeliefert wurden, wird hinfällig bei einem Blick' auf unsere Krankengeschichten, in denen sich fast durchweg die Angabe findet, dass 
Kranken in schwer ausgeblutetem Zustande in die Klinik eingeliefert wurden.

Wenn wir demnach die Qualität des Materials als Konstante in Rechnung setzen dürfen, dann gibt es nur eine Erklärung für die Verschiedenheit der Resultate in dem Winckel'schen und Döderlein'schen Materiale, nämlich die Verschiedenheit des therapeutischen Vorgehens in den beiden Vergleichsserien.

Bei der Wertung der versehiedenen Behandlungsmethoden ist zunächst zu erwägen, dass gewisse, allgemein anerkannte Eingriffe - nämlich die künstliche Blasensprengung und die rechtzeitige innere Wendung - sich in beiden Serien finden müssen. Da bei beiden Methoden die Vorbedingungen und Indikationen scharf umrissen sind und da auch die technische Ausführung keire wesentlichen Modifikationen ermöglicht, so ist von vornherein zu. erwarten, dass die Resultate in beiden Serien im grossen und ganzen gleich sind. Dies ist nun in der Tat auch der Fall. So verlor v. Winckel unter seinen Fällen von Blasensprengung keine Mutter, Döderlein eine einzige, und für die rechtzeitige innere Wendung ergibt sich ans dem Winckel'schen Material eine mütterliche Mortalität von $24 \mathrm{pCt}$, aus dem Döderlein'schen eine von $16 \mathrm{pCt}$.

Das Anwendungsgebiet der Blasensprengung und rechtzeitigen inneren Wendung ist aber nur ein recht begrenztes und enges. Zur Wertung der eigentlichen Resultate kommt daher nur das Gros der Placenta praevia-Fälle in Betracht, die auf eine andere Weise behandelt wurden. Ausser der Blasensprengung und der direkten Wendung finden sich nun in dem Winckel'schen Material als Behandlungsmethoden der Placenta praevia-Fälle erwähnt: 1. nur Tamponade, 2. Tamponade mit Blasensprengung, 3. kombinierte Wendung mit Extraktion, 4. kombinierte Wendung ohne Extraktion, 5. innere Wendung ohne Extraktion, 6. Herabholen eines Fusses ohne Extraktion, 7. Herabholen eines Fusses mit Extraktion.

Die mütterliche Mortalität aller dieser Eingriffe beträgt $14,6 \mathrm{pCt}$.

Dieser Vielheit von Methoden steht in dem Döderlein'sehen Materiale als das Verfahren der Wahl einzig und allein der vaginale Kaiserschnitt gegenüber mit einer Mortalität von 9,6 pCt.

Die in dem Gesamtmateriale schon vorhandene Differenz in der mütterlichen Mortalität zwischen den Fällen v. Winckel's und 
Döderlein's bleibt also auch bei Einengung auf die eigentlich ausschlaggebenden Operationsmethoden bestehen. Mit anderen Worten, durch die Einführung des vaginalen Kaiserschnittes in die Placenta praevia-Behandlung ist es an der Münchener Universitäts-Frauenklinik gelungen, die mütterliche Mortalität auf die Hälfte ihres früheren Wertes zu reduzieren.

Neben den eigentlichen - therapeutischen Eingriffen spielen unseres Erachtens in der Placenta praevia-Therapie auch die

\section{Vor- und Nachbehandiung}

eine gewisse, durchaus nicht zu unterschätzende Rolle. Sicher ist. schon der Zeitpunkt des operativen Eingriffes nicht ohne Bedeutung. So halten wir nicht für richtig, wenn man jeden Fall von. Placenta praevia - vorausgesetzt, dass die ganze Sachlage nicht dazu drängt - direkt von der Tragbahre des Sanitätswagens auf den Operationstisch legt. Der vorausgegangene Blutverlust, die von den Aerzten und Hebammen vorgenommenen Eingriffe, die körperlichen und seelischen Alterationen beim Transport in die Klinik; alle diese Momente üben eine gewisse Shockwirkung auf die Kranke aus. Diese Wirkung ist nun zwar eine inkommensurable Grösse. Dass sie aber ein tatsächlich vorhandener und in Rechnung zu setzender Faktor ist, der von unbestreitbarem Einfluss auf die Prognose operativer Eingriffe sein kann, das hat uns der Krieg wieder eindringlich an unseren Verwundeten gezeigt. Die. Döderlein'sche Klinik hat daher von jeher bei allen den Fällen von Placenta praevia, die kein direktes Eingreifen erforderten, Wert auf eine gewisse Vorbehandlung der Frauen gelegt. Diese erstrebt vor allem eine. körperliche und seelische Kräftigung. Nicht durch die in diesem Stadium der Geburt noch schädlichen und aufs strengste verpönten Analeptika, sondern vor allem durch Ruhe und Flüssigkeitszufuhr. Hierdurch wird die posthämorrhagische Reparationstätigkeit der blutbildenden Organe unterstützt, durch die Füllung des Gefässsystems werden vasomotorische Druckausschläge verhindert oder. wenigstens verkleinert, die psychische Akkommodation der Kranken an das neue Milieu erleichtert neben prophylaktischen Morphium-Skopolamininjektionen die eventuell nötig werdende Narkose. Ein weiterer und für den späteren Verlauf durchaus nicht zu vernachlässigender Faktor ist die Möglichkeit einer ausgiebigen Entleerung des Darmes. 
Während der entbindenden Operation selbst empfiehlt es sich zur leichteren Beherrschung der Blutungen unter die Lendenwirbelsäule der Patientinnen den Momburg'schen Schlauch oder die Rückenplatte der Gauss'schen Aderpresse zu legen, damit man im Notfall, wenn eine der beiden Methoden zur Anwendung kommen soll, keine unnötige Zeit verliert. Ferner hat sich uns auch das Abschnüren der Beine sehr bewährt. Wir machen es derart, dass wir hoch oben, an den Oberschenkeln, je eine Esmarch'sche Binde so anlegen, dass wohl das arterielle Blut noch in die beiden unteren Extremitäten gelangen kann, dass aber der venöse Rückfluss vollkommen oder wenigstens fast vollkommen verhindert wird. Auf diese Weise erhalten wir in beiden Beinen durch die starke venöse Stauung je ein ziemlich grosses Blutreservoir. Werden nun nach Beendigung der Operation und sicherer Blatstillung die Binden von den beiden Oberschenkeln abgenommen, dann kann das in den beiden Beinen arretierte Blut wieder in den allgemeinen Kreislauf übertreten. Diese physiologische Autotransfusion körpereigenen Blutes ist so gut wir immer von deutlichem Einfluss auf das Befinden der Patientin. Man ist oft überraseht, wie auffallend sich daraufhin das subjektive und objektive Befinden hebt.

Auch die Nachbehandlung der wegen Placenta praevia Operierten ist sebr verantwortungsvoll und keineswegs leicht. Ein starres schematisches Anwenden der für die Behandlung grosser Blutverluste gegebenen Vorschriften kann den ganzen Effekt der Operation vereiteln und schliesslich doch noch einen deletären Ausgang herbeiführen.

Zunächst muss man auch nach der völligen Entbindung und Blutstillung mit der Anwendung von Exzitantien sehr vorsichtig sein. Die Erhöhung des Blutdruckes nach Anwendung von Kampfer, Koffein usw. kann die Blutung wieder von neuem beginnen lassen und es ist dann durchaus nicht unmöglich, dass sich die Kranke noch nachträglich in ihre Tamponade hinein verblutet. Man muss sich stets vor Augen halten, dass im puerperalen Uterus und speziell in dem funktionell minderwertigen Isthmus die Blutstillung eine ganz andere ist, als die nach chirurgischen Operationen.

Neben der Anwendung von Exzitantien erscheint als das gegebene Verfahren die möglichst rasche Ersetzung des verloren gegangenen Blutes. Zu diesem Zweck wird allgemein die subkutane 
oder intravenöse Kochsalzinfusion empfohlen. Von ihr sind wir in der Behandlung der puerperalen Blutungen seit einiger Zeit abgekommen. Der Grund hierfür waren Beobachtungen, die wir bei der Sektion verbluteter Frauen machten, die eine Kochsalzinfusion erhalten hatten. Es fanden sich in diesen Fällen nämlich eigenartige Veränderungen vor allem an Herz, Leber, Nieren und Gehirn, und zwar waren es in erster Linie die Zeichen von trüber Schwellung und Oedem, die uns auffielen und die so charakteristisch waren, dass man aus ihnen geradezu intra vitam vorgenommene Kochsalzinfusion diagnostizieren konnte.

Ueber die Genese dieser Veränderungen konnten wir natürlich nur Vermutungen äussern, später fanden wir eine Reihe hierher gehöriger Tatsachen in der interessanten Arbeit von Thies ${ }^{1}$ ).

Jedenfalls erscheint uns die physiologische Kochsalzlösung als ein sehr differentes Mittel, dessen chemisch-physikalische Wirkungen auf die verschiedenen Körperzellen durchaus noch nicht geklärt sind.

So sind wir ohne jede Kenntnis der Arbeit von Thies ganz von selbst dazu gekommen, an die Stelle von Kochsalzinfusionen rektale Tropfeinläufe von gewöhnlichem Wasser, eventuell mit etwas Zusatz von Adrenalin zu geben. Daneben legen wir grossen Wert darauf, dass den Kranken sobald als möglich per os in regelmässigen kurzen Intervallen kleine Flüssigkeitsmengen zugeführt werden. Exzitantien geben wir nur in Ausnahmefällen bei plötzlichem Kollaps, auch rom Fatschen der Beine und der Anwendung der Lichtbögen sind wir - in der Behandlung puerperaler Blutungen - abgekommen. Mit diesem, im Vergleich zu der sonst üblichen Polypragmasie recht einfachen therapeutischen Apparat haben wir in der letzten Zeit ganz ausgezeichnete Érgebnisse erzielt.

"De tous les accouchements celui-là est le plus hazardeux" sagte Pierre Dionis von der vorliegenden Nachgeburt. Dass auch heute noch ein guter Ausgang bei Placenta praevia mehr oder weniger Glückssache ist, das zeigen die 20 pCt. Mortalität im Privathause, wo jede 5. Frau mit Placenta praevia dieser Komplikation erliegt. Eine Placenta praevia-Kranke hat nach diesen Resultaten ungefähr die gleichen Chancen, mit dem Leben davon

1) Mitteil. a. d. Grenzgeb. usw. Bd, 21. S. 239. 
zu kommen, als wenn sie die Cholera hätte, während vom klinischen Standpunkt aus die Erkrankung nur einer leichten Ruhr ${ }^{1}$ ) entspricht.

Unter diesen Umständen ist es unverständlich, wie beim Auftreten dieser sehweren Dystokie Aerzte und Laien überhaupt nur eine Minute über den einzuschlagenden Weg im Unklaren sein können.

„Ueber das, was richtig ist, darf keine andere Rücksicht entscheidend sein als das Wohl der Kranken. Wenn die Mögliehkeit sich herausstellt, dass durch eine operative Behandlung mit dem Messer mehr Mütter und Kinder am Leben zu erhalten sind als mit den im Privathause möglichen Eingriffen, so muss jede andere Rücksicht zurücktreten. Dann wird es für die alleinstehenden, zu grossen Operationen nicht eingerichteten und nicht ausgebildeten Aerzte zur Pflicht, diejenigen Fälle von Placenta praevia, die schon durch eine starke Blutung in der Schwangerschaft oder im Anfang der Geburt bei engem Muttermund und mangelhaften Wehen sich kenntlich machen, in eine entsprechende Krankenanstalt $\mathrm{zu}$ schicken." [Z $\left.\mathrm{Weifel}^{2}\right)$.]

1) Nach P. Th. Müller (Vorlesungen über Infektion und Immunität, Jena 1917, S. 414) betrug die Cholerasterblichkeit — allerdings der einmal Geimpften - bei der österreichisch-ungarischen Armee in den Jahren 1914 bis 191526 Ct. Nach Jochmann (Mohr und Staehelin, Handb. d. inn. Med., 1911, Bd. 1, S. 348) beträgt die Sterblichlkeit der Shiga-Kruse-Dysenterie $10-15 \mathrm{pCt}$, die der übrigen Bazillenruhrformen bis zu $5 \mathrm{pCt}$.

2) Döderlein, Handb. Bd. 2. S. 615. 